2008s-25

\title{
Régulation et mode de gestion : une étude économétrique sur les prix et la performance dans le secteur de l'eau potable
}

\author{
Marcel Boyer, Serge Garcia
}

\section{Série Scientifique \\ Scientific Series}

Montréal

Septembre 2008

(C) 2008 Marcel Boyer, Serge Garcia. Tous droits réservés. All rights reserved. Reproduction partielle permise avec citation du document source, incluant la notice (C).

Short sections may be quoted without explicit permission, if full credit, including (C) notice, is given to the source.
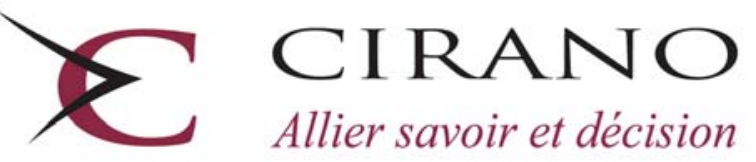

Allier savoir et décision

Centre interuniversitaire de recherche en analyse des organisations 


\section{CIRANO}

Le CIRANO est un organisme sans but lucratif constitué en vertu de la Loi des compagnies du Québec. Le financement de son infrastructure et de ses activités de recherche provient des cotisations de ses organisations-membres, d'une subvention d’infrastructure du Ministère du Développement économique et régional et de la Recherche, de même que des subventions et mandats obtenus par ses équipes de recherche.

CIRANO is a private non-profit organization incorporated under the Québec Companies Act. Its infrastructure and research activities are funded through fees paid by member organizations, an infrastructure grant from the Ministère du Développement économique et régional et de la Recherche, and grants and research mandates obtained by its research teams.

\section{Les partenaires du CIRANO}

\section{Partenaire majeur}

Ministère du Développement économique, de l’Innovation et de l’Exportation

\section{Partenaires corporatifs}

Alcan inc.

Banque de développement du Canada

Banque du Canada

Banque Laurentienne du Canada

Banque Nationale du Canada

Banque Royale du Canada

Banque Scotia

Bell Canada

BMO Groupe financier

Bourse de Montréal

Caisse de dépôt et placement du Québec

DMR Conseil

Fédération des caisses Desjardins du Québec

Gaz de France

Gaz Métro

Hydro-Québec

Industrie Canada

Investissements PSP

Ministère des Finances du Québec

Raymond Chabot Grant Thornton

State Street Global Advisors

Transat A.T.

Ville de Montréal

\section{Partenaires universitaires}

École Polytechnique de Montréal

HEC Montréal

McGill University

Université Concordia

Université de Montréal

Université de Sherbrooke

Université du Québec

Université du Québec à Montréal

Université Laval

Le CIRANO collabore avec de nombreux centres et chaires de recherche universitaires dont on peut consulter la liste sur son site web.

Les cahiers de la série scientifique (CS) visent à rendre accessibles des résultats de recherche effectuée au CIRANO afin de susciter échanges et commentaires. Ces cahiers sont écrits dans le style des publications scientifiques. Les idées et les opinions émises sont sous l'unique responsabilité des auteurs et ne représentent pas nécessairement les positions du CIRANO ou de ses partenaires.

This paper presents research carried out at CIRANO and aims at encouraging discussion and comment. The observations and viewpoints expressed are the sole responsibility of the authors. They do not necessarily represent positions of CIRANO or its partners. 


\title{
Régulation et mode de gestion : une étude économétrique sur les prix et la performance dans le secteur de l'eau potable
}

\author{
Marcel Boyer ${ }^{\dagger}$, Serge Garcia ${ }^{\ddagger}$
}

\begin{abstract}
Résumé / Abstract
Nous modélisons les interactions entre mode de gestion (régie ou délégation) et coûts d'exploitation des services d'eau potable afin de comparer leurs performances et la tarification. Nous estimons ces modèles sur des données de panel en France. Nous montrons que le choix de la collectivité locale dépend des coûts et des caractéristiques des services. Il existe une différence significative d'efficacité productive moyenne en faveur de la gestion déléguée, mais les services en régie semblent plus performants sur les rendements de réseau. Les marges réalisées par l'exploitant sont expliquées par les caractéristiques des services, et celles du contrat dans le cas de délégation.
\end{abstract}

Mots clés : services d'eau potable, mode de gestion, modèle de sélection, fonction de coût, tarification, données de panel.

We model the interactions between management regimes (municipal vs. delegated) and operating costs of water supply services in order to compare their performance and pricing. We estimate the models from panel data in France. We show that the choice between management regimes at the local community level depends on costs and service characteristics, that there is a significant difference in average productive efficiency in favor of delegated services, and that municipal services appear more efficient in network returns. Under delegation, the margins realized by operators depend on service and contract characteristics.

Keywords: water supply services, management regime, selection model, cost function, pricing, panel data.

Codes JEL : C25, C33, D42, L25, L95

\footnotetext{
* Les auteurs remercient les deux rapporteurs anonymes choisis par la revue, ainsi que Bruno Crépon et Sébastien Roux les rédacteurs en chef, pour leurs commentaires constructifs. Ce papier a bénéficié également des remarques d'Alain Carpentier et Patrick Sevestre, et aussi des participants aux journées AFSE (Rennes, 2004) et aux journées de microéconomie appliquée (Lille, 2004). Merci également à Lætitia Guérin Schneider ainsi qu'aux participants du Séminaire SEGEPRE (Montpellier, 2003) pour leurs réactions sur une première version de cet article. Les auteurs sont reconnaissants à D. Careil (DDAF), F. Regimbal (ESG) et le directeur d'une grande structure de distribution d'eau potable du Nord de la France pour la fourniture des données. Des versions préliminaires de cet article ont été rédigées pendant que le second auteur était accueilli par le laboratoire CIRANO pour un stage postdoctoral puis au laboratoire GEA-ENGREF.

À paraître dans Annales d'économie et de statistique.

† Titulaire de la Chaire Bell Canada en économie industrielle, département de sciences économiques, Université de Montréal, C.P. 6128, succursale Centre-ville, Montréal (Québec), H3C 3J7, Canada, et Fellow CIRANO, e-mail :

marcel.boyer@cirano.qc.ca, tél : 1-514-985-4002.

† INRA, UMR 356 Économie Forestière, F-54000 Nancy, France, et Agroparistech, Engref, Laboratoire d'Économie Forestière, F-54000 Nancy, France. Adresse : LEF, INRA-ENGREF, 14 rue Girardet, CS 14216, Nancy, 54042 France. Email : garcia@nancy-engref.inra.fr, tél : 03-83-39-68-69.
} 


\section{Introduction}

Les services d'eau sont des monopoles naturels locaux. En France, l'organisation des services publics locaux incombe aux communes. Les services d'alimentation en eau potable (AEP) et d'assainissement peuvent être gérés directement par la commune (en régie) ou bien faire l'objet d'une délégation de service public (à une entreprise privée). Dans ce contexte, une tarification au coût marginal entraîne un déficit budgétaire. Dans le cas d'une gestion publique, une solution serait de maximiser le bien-être social sous contrainte d'équilibre budgétaire. Lorsque la gestion est déléguée, l'entreprise va chercher à fixer un prix au-dessus du coût marginal et la collectivité à réduire la marge de l'entreprise tout en l'incitant à fournir le meilleur service ${ }^{1}$. Pour ces raisons, la procédure de délégation passe par un appel d'offres. DEMSETz [1968] préconise la concurrence pour le marché comme complément voire substitut de la régulation du monopole. RIORDAN et Sappington [1987] modélisent la procédure optimale d'attribution de la concession d'un monopole lorsque les producteurs potentiels ont une information privée sur les coûts. MOUGEOT ET NAEGELEN [2005] reformulent cette théorie en faisant le lien entre régulation du monopole à la Baron-Myerson et procédure de mise en concurrence pour le marché2.

Notre article s'inscrit dans ce cadre en caractérisant le mécanisme incitatif optimal avec asymétries dans la distribution de l'information lorsque la gestion de l'eau est déléguée. Quelques études économétriques s'intéressent aux problèmes informationnels dans le secteur de l'eau. L'article pionnier de WOLAK [1994] étudie le secteur de l'eau potable en Californie à partir d'un modèle d'information privée et montre que l'estimation des coûts est meilleure que dans un modèle avec information complète. Les travaux de Garcia et ThOmas [2003] en France et ceux de BrocAs et al. [2006] aux États-Unis mettent aussi en évidence les distorsions dues aux asymétries d'information.

Notre travail a comme objectif supplémentaire de comparer les coûts, la performance et les prix entre les modes de gestion (régie vs. délégation). Il ne suffit pas d'estimer le différentiel des coûts ou des prix en utilisant une variable binaire indiquant le mode de gestion choisi par la commune, ni d'estimer séparément les équations de coût ou de prix pour chaque mode de gestion. S'il existe différents modes de gestion offrant chacun des avantages différents pour la commune, le choix d'un

\footnotetext{
${ }^{1}$ On parle de rente informationnelle car elle est liée au déficit d'information de la collectivité sur l'entreprise et/ou sur le réseau. Le problème peut également se poser dans la cas d'une gestion directe où les services techniques de la commune dissimuleraient, sciemment ou non, au conseil municipal certaines informations ou certaines de leurs actions sur le plan de production afin de profiter de rentes de situation.

${ }^{2}$ BAron et Myerson [1982] considèrent le cas où le régulateur (ici la collectivité locale) n'a pas une information complète sur les coûts mais en connaît ou peut en estimer la distribution. Le régulateur définit un mécanisme optimal en maximisant l'espérance d'une fonction linéaire de l'utilité des usagers et des profits de l'entreprise sous contraintes de participation et d'incitation de l'entreprise. Pour inciter l'entreprise monopole à révéler ses coûts tout en évitant de lui verser tout le surplus, le régulateur crée une distorsion (optimale) dans la demande exprimée et ce faisant accepte une certaine perte (minimale) d'efficacité.
} 
mode plutôt qu'un autre sera déterminé par un certain nombre de facteurs en plus du différentiel de coûts. Ignorer l'endogénéité de ce choix conduirait à un biais de sélection lors de l'estimation des équations de coût ou de prix pour les services en régie et ceux en gestion déléguée.

La possibilité de choisir le mode de gestion des services d'eau et les différences de prix constatées d'une commune à l'autre ont donné matière à de nombreux débats sur l'organisation des services d'eau. Mais seulement quelques études ont tenté d'expliquer le choix de mode de gestion des services d'eau en France et son impact sur les prix ${ }^{3}$. SAGE [1999] montre que le mode de gestion n'explique pas à lui seul les différences de prix de l'eau et que la comparaison directe des prix ne peut pas être satisfaisante. D'autres études sur les services d'eau français (MÉNARD et SAUssier, [2000]; ChOng et al., [2006]; CARPEnTier et al., [2006]) se rejoignent sur ce constat et concluent qu'il n'existe pas d'avantage absolu d'un mode de gestion sur l'autre et que la performance et le choix de mode de gestion dépendent de facteurs observés et non observés.

Dans notre article, nous utilisons des données de panel sur les coûts d'exploitation et les prix de services d'AEP en régie et en délégation en France. L'assainissement qui constitue une part importante du prix de l'eau mais qui est un service indépendant de l'AEP, est exclu du périmètre de notre étude. Un premier travail consiste à modéliser le choix de mode de gestion qui est déterminé par la différence des coûts, les caractéristiques des services et le mode de gestion de l'assainissement. Nous utilisons des méthodes d'estimation dérivées des travaux de HeCKMAN [1976, 1979] et de LEE [1978] pour traiter l'éventuel biais de sélection, adaptées aux données de panel. Nous estimons d'abord le modèle Probit de choix de mode de gestion qui se fait à une période donnée de façon définitive. Les coûts, observés sur plusieurs périodes, sont ensuite régressés sur les déterminants du choix ainsi que sur les ratios de Mills inverses issus de la première étape pour corriger le biais de sélection. Les méthodes d'estimation utilisées exploitent les spécificités des données de panel -introduction d'hétérogénéité non observée (effets individuels) et possibilité de corrélation avec les régresseurs. Le modèle complet est identifié de façon non paramétrique en excluant la variable représentant le mode de gestion de l'assainissement puisque celui-ci n'a pas d'impact sur les coûts d'AEP. Nous pouvons alors calculer l'efficacité productive et les rendements de réseau des services, et comparer les résultats selon le mode de gestion ${ }^{4}$.

Le second travail est la modélisation de la tarification pour chaque mode de gestion. Ce modèle

\footnotetext{
${ }^{3}$ Reynaud et Thomas [2005] montrent que des facteurs déterminant le choix de mode de gestion conditionnent aussi la consommation d'eau des usagers domestiques.

${ }^{4}$ Avec l'augmentation des coûts de service ces dernières années en raison de la complexification et la spécialisation du métier, la performance comparée des modes de gestion est devenue une préoccupation croissante pour les décideurs politiques et certaines institutions internationales comme la Banque mondiale (ESTACHE et RosSI, [2002]). En France, un ensemble d'indicateurs de performance utilisés par les collectivités locales a été développé pour contrôler la qualité du service (GuÉRIN-SCHNEIDER et NAKHLA, [2003]). Ils peuvent être utilisés afin de comparer plusieurs services dans le cadre de participation volontaire à un réseau de benchmarking ou bien d'un système obligatoire de concurrence par comparaison (yardstick competition).
} 
est construit, pour les régies, sur un modèle de régulation Cost of service. Cela conduit à la formule de l'indice de Lerner. En gestion déléguée, la collectivité locale organise un appel d'offres pour la gestion du service par une entreprise extérieure et le mécanisme optimal aboutit à un prix BaronMyerson. Dans ce cas, c'est la différence prix-coût marginal qui est estimée. Ces deux modèles sont estimés sur données de panel en corrigeant les biais de sélection. Les résultats montrent que les caractéristiques des services expliquent les variations de marges observées et nous permettent, dans le cas de gestion déléguée, de calculer le niveau moyen des rentes informationnelles.

La suite de l'article est organisée de la façon suivante. La section 2 présente brièvement l'organisation et la réglementation des services d'eau en France, ainsi que les caractéristiques de la technologie afin de spécifier la fonction de coût variable utilisée dans le modèle économique. Nous décrivons ensuite les bases de données de services d'AEP en régie et en délégation. Le modèle de choix de mode de gestion des services est exposé dans la section 3. La section 4 est consacrée à la présentation de la procédure d'estimation et des résultats d'estimation concernant le choix de mode de gestion et les coûts. Nous présentons enfin l'étude sur la tarification dans la section 5 . La dernière section conclut notre travail.

\section{Les services d'eau en France}

\subsection{Organisation et réglementation}

En France, les services d'eau (AEP et assainissement) peuvent être gérés directement en régie (avec autonomie financière ou/et personnalité morale) ou bien faire l'objet d'un contrat de délégation (affermage ou concession) avec une entreprise privée ${ }^{5}$. De tels contrats spécifient la nature des services attendus, le tarif payé par les usagers et, dans le cas des contrats d'affermage, la part des travaux de renouvellement incombant à l'opérateur. Le contrat d'affermage est la forme la plus répandue en France (pour l'AEP, 88\% de la population en gestion déléguée ${ }^{6}$ ), car c'est la formule la plus souple permettant de s'adapter à un grand nombre des situations. Il est signé en général pour une durée de 7 à 12 ans. L'opérateur a la responsabilité de l'exploitation et la maintenance du réseau, il facture lui-même le service et redistribue la part revenant à la collectivité.

Quel que soit le mode de gestion, les services d'eau font face au même environnement réglementaire. C'est dans les années 1990 que l'évolution de la réglementation est déterminante. La loi sur l'eau de 1992 réactualise le cadre législatif mis en place en 1964. La loi "Sapin" de 1993 a pour objectif de mettre un terme aux pratiques illicites entre entreprises privées et responsables

\footnotetext{
${ }^{5}$ En France, l'exploitation des services d'eau par des entreprises publiques de statuts de droit privé est rare contrairement à d'autres pays de l'Union européenne comme l'Allemagne ou les Pays-Bas.

${ }^{6}$ Direction de l'eau au Ministère de l'aménagement du territoire et de l'environnement, chiffres repris par le rapport d'information à l'Assemblée nationale sur le financement et la gestion de l'eau (TAVERNIER, [2001]).
} 
des collectivités locales ${ }^{7}$ en introduisant des procédures de publicité et de mise en concurrence des contrats de délégation (concurrence pour le marché). Cependant, il n'existe pas d'autorités administratives et financières centrales pour régir les règles économiques que devraient respecter les entreprises en charge des services publics de l'eau. Ce sont les collectivités délégantes qui sont responsables de la régulation et du contrôle des services, aidés si nécessaire par les $\mathrm{DDAF}^{8}$ ou par un conseil privé (cabinets d'audit).

Par ailleurs, les services d'eau doivent respecter les principes de service public, dont le principe d'égalité de traitement des usagers concernant l'accès au service, les prestations et le tarif du service $^{9}$. De plus, les services d'eau sont des services publics industriels et commerciaux (SPIC) leur imposant des règles particulières tant sur le plan juridique qu'en matière budgétaire et de gestion de trésorerie. Le nouveau droit de l'eau issu de la législation de 1992 et les réglementations qui ont suivi reflètent la volonté des pouvoirs publics de mieux tenir compte des réalités économiques des services. Cela implique par exemple la mise en place de services d'eau financièrement autonomes, c'est-à-dire présentant un budget équilibré et financé par l'usager. En outre, les services d'eau des collectivités de plus de 3000 habitants ont l'obligation de tenir un budget spécifique au service, propre ou annexe du budget général de la collectivité et la séparation entre AEP et assainissement doit être claire sur la facture de l'eau ${ }^{10}$. Les autres contraintes réglementaires portent sur la qualité de l'eau ${ }^{11}$. La réglementation environnementale affecte les services d'eau à travers son impact sur le traitement de l'eau et les coûts de réduction d'effluents d'eaux usées, et de manière identique lorsque les conditions environnementales sont homogènes.

En gestion directe, le prix de l'eau est le résultat d'une délibération du conseil municipal ou syndical de la collectivité locale. L'objectif principal est d'atteindre l'équilibre budgétaire. Lorsque le service est délégué, le prix est déterminé suite au résultat de l'appel d'offres. Une nouvelle phase de négociation (principe de l'intuitu personae) permet à la collectivité et l'opérateur d'ajuster le prix et la qualité de service. Le prix est établi à partir d'un compte d'exploitation prévisionnel fourni par l'opérateur. Le délégataire présente le plus souvent des marges prévisionnelles quasi nulles (CANNEvA et al., [2004]), de sorte que la collectivité peut croire à un remboursement des coûts. Cependant, les coûts ne sont que très rarement contrôlés, laissant les problèmes d'informa-

\footnotetext{
${ }^{7}$ On pense par exemple à l'affaire de corruption mise à jour dans la concession des services d'eau de Grenoble.

${ }^{8}$ Les DDAF (Directions Départementales de l'Agriculture et de la Forêt) peuvent être chargées du suivi des délégations des services ou de la reconstitution des coûts du service lors d'une négociation de contrat.

${ }^{9}$ Le principe de continuité donne aux usagers un droit d'accès normal et permanent au service, et le principe d'adaptation requiert l'adaptation du service à l'évolution des besoins des usagers.

${ }^{10}$ Loi Barnier 1995, Titre V, Art. 74.

${ }^{11}$ Deux directives édictées par la Commission européenne concernent la qualité des eaux de surface pour la production d'eau potable et la qualité des eaux destinées à la consommation humaine. Cette dernière définit les concentrations maximales pour 65 paramètres de qualité de l'eau potable. Les agences de l'eau en France ont adopté une politique de protection de la ressource basée sur les redevances de prélèvement et de pollution. Les revenus de ces taxes sont redistribués entre pollueurs et collectivités locales sous la forme d'aides financières dans le but d'investir dans des infrastructures comme des stations d'épuration.
} 
tion privée non résolus. Une formule d'actualisation permet d'établir l'évolution du prix chaque année et généralement pour cinq ans. Cette formule prévoit un ajustement du prix en fonction de l'évolution du coût des principaux facteurs de production ${ }^{12}$. Quel que soit le mode de gestion, un montant (appelé part fixe ou abonnement) indépendant du volume d'eau consommé peut être facturé. Selon l'article 13-II de la loi sur l'eau de 1992, l'abonnement doit tenir compte des charges fixes du service et des caractéristiques du branchement. Cette définition laconique rend sa détermination délicate et laisse une marge de manœuvre non négligeable aux exploitants ${ }^{13}$.

Le choix de mode gestion des services d'eau se traduit par une différence de prix significative. Selon la Direction Générale de la Concurrence, de la Consommation et de la Répression des Fraudes, en 1998 le prix moyen des services d'eau en régie était de $13 \%$ inférieur à celui des services délégués. Une enquête de l'Institut français de l'environnement sur la même année révèle une différence de prix plus marquée de l'ordre de 27\% pour l'AEP et 20,5\% pour l'assainissement (IFEN, [2001]). L'écart s'élève même à $44 \%$ entre les régies communales et les syndicats intercommunaux en affermage. Dans son rapport, TAVERNIER [2001] avance que cette différence entre les deux études provient de la nature des échantillons (urbain vs. rural). Les arguments des délégataires pour expliquer ces écarts sont nombreux (taxes, investissements, difficultés des services), mais les avantages de la gestion par des opérateurs privés existent (économies d'échelle liées à la gestion de plusieurs contrats, possibilité de placements de trésorerie). Le rapport rappelle aussi l'existence d'autres facteurs ayant un impact sur le prix tels que la qualité du service, les asymétries d'information au dépens des collectivités et des usagers, le manque de clarté des contrats, le manque d'expertise des collectivités et les contrôles insuffisants. Par ailleurs, selon la Cour des comptes ${ }^{14}$, les régies ont des rendements médiocres (beaucoup de personnel insuffisamment qualifié, mauvais état des réseaux, absence de rigueur dans la gestion et la facturation).

\footnotetext{
${ }^{12}$ Chaque poste de dépenses est pondéré par un coefficient (différent d'un contrat à l'autre) de manière à refléter au mieux l'environnement économique du service. La formule d'actualisation du prix de l'eau est du type suivant : $P=P_{0} \times\left(k+a \frac{A}{A_{0}}+b \frac{B}{B_{0}}+\ldots\right)$, avec $k+a+b+\ldots=1$. $P$ est le prix à appliquer chaque année et $P_{0}$ le prix de début de contrat. $k$ est une constante (supérieure ou égale à 0,1 ), $a$ et $b$ sont les coefficients associés aux ratios $\frac{A}{A_{0}}$, $\frac{B}{B_{0}}$ représentant les variations de coût des facteurs (indice 0 pour l'année initiale du contrat).

${ }^{13} \mathrm{~L}$ 'Office international de l'eau souligne que les montants des abonnements peuvent atteindre parfois $150 € /$ an et intègrent de toute évidence d'autres éléments, entretenant ainsi une certaine opacité des comptes.

${ }^{14}$ Voir les rapports publics sur la gestion des services publics d'eau et d'assainissement (COUR DES COMPTES, [1997, 2003]).
} 


\subsection{Technologie}

Le secteur de l'eau ${ }^{15}$ se présente comme un marché de nature monopolistique au moins pour une partie de ses activités. L'AEP peut être séparée en deux activités : la production d'eau potable proprement dite et la distribution de cette eau vers différentes catégories d'usagers. La distribution de l'eau potable engendrent des coûts fixes importants. La spécificité des actifs donne à ces coûts fixes un caractère irréversible. De plus, la construction de réseaux parallèles est tout à fait irréaliste et constitue une véritable barrière à l'entrée pour un concurrent éventuel, ce qui confère à l'activité de distribution une structure monopolistique non contestable. Par ailleurs, l'eau est un produit lourd dont le transport est coûteux et le stockage difficile, et les exigences de qualité nécessitent une certaine proximité entre les lieux de production et de consommation. L'AEP est donc un service qui a naturellement incombé aux collectivités locales en France et ailleurs.

Les objectifs généraux du service d'eau potable sont de produire une eau de bonne qualité à partir d'une eau brute pouvant nécessiter un traitement et de la mettre à disposition des usagers en s'adaptant en permanence à leur demande et en préservant la qualité de l'eau durant son séjour dans le réseau. Les coûts d'exploitation du service sont donc engendrés par toutes les opérations ayant trait à la desserte en eau potable depuis le prélèvement dans le milieu naturel jusqu'au robinet de l'usager ${ }^{16}$. L'environnement dans lequel le service évolue explique en grande partie les différences de coûts (et de prix) observées entre différents réseaux d'eau. Par exemple, l'eau potable peut être produite à partir d'eaux brutes souterraines ou de surface. L'eau issue d'une nappe souterraine engendre des coûts plus importants de forage et de pompage alors que les coûts de traitement sont d'ordinaire plus conséquents pour les eaux de surface. Par ailleurs, les coûts variables peuvent être également très différents lors de la phase de distribution, car ils dépendent de façon cruciale de la taille des zones desservies, leur densité en population et la topographie.

Une partie du volume d'eau mis en distribution dans le réseau n'atteint pas sa destination finale, principalement à cause de pertes dues à des ruptures de conduites ou des joints fuyants ${ }^{17}$. Cette spécificité doit être prise en compte car les coûts de production et de distribution dépendent fortement de l'état du réseau. Le rendement de réseau calculé comme le ratio du volume facturé aux usagers et du volume mis en distribution est un indicateur important pour les ingénieurs et

\footnotetext{
${ }^{15}$ Le secteur de l'eau possède des caractéristiques similaires à celles d'autres activités industrielles en réseau (électricité, télécommunications, transports) : structure monopolistique du marché, prédominance de l'opérateur historique, obligations de service public... Mais en dépit de ces caractéristiques communes, il existe des différences importantes entre les secteurs : progrès technologiques, conditions de demande, globalisation des marchés (franchissement transfrontalier dans le secteur des transports, présence de grands groupes transnationaux dans les secteur de l'eau et l'électricité par exemple), certains segments de l'industrie sont des monopoles naturels et d'autres plus concurrentiels etc.

${ }^{16}$ Voir Garcia et Thomas [2001] pour une présentation plus détaillée de cette technologie.

${ }^{17}$ Lors de l'étape de production, les conduites de transfert étant peu nombreuses, les fuites sont facilement localisables et donc rapidement réparées.
} 
une variable de décision cruciale pour les gestionnaires du service. Garcia et Thomas [2001] soulignent l'existence d'économies de coût substantielles selon que l'exploitant choisit d'accroitre la production d'eau potable tout en maintenant l'état du réseau inchangé ou bien de réparer les fuites sans extraire davantage d'eau. Par ailleurs, Garcia et Thomas [2003] montrent qu'en raison de l'information incomplète sur les coûts de production d'eau potable et de maintenance du réseau de distribution lorsque la collectivité locale délègue l'exploitation du service public à un opérateur privé, elle doit autoriser ce dernier à "produire" un niveau de pertes d'eau supérieur au niveau optimal d'information complète.

\subsection{Modélisation des coûts}

La modélisation et l'estimation de fonctions de coût ainsi que l'étude de l'effet du type de propriété (public ou privé) des services d'eau sur leur efficacité sont des sujets qui ont été traités par un certain nombre d'articles de recherche empirique depuis plusieurs années ${ }^{18}$. EsTACHE et Rossi [2002] présentent les estimations de frontières de coût dans le but de mesurer l'efficacité productive des compagnies publiques et privées chargées de la distribution de l'eau potable dans la région d'Asie et du Pacifique. Ils font notamment un bref survol des travaux réalisés à ce sujet dans le secteur de l'eau. Par ailleurs, SAAL et PARKER [2000] étudient l'impact de la privatisation et de la régulation économique et environnementale sur la performance économique des services d'eau en Grande-Bretagne à partir d'une fonction de coût translog multi-produits (AEP et assainissement).

Les études réalisées sur le sujet et la description de la technologie faite plus haut nous permettent d'identifier plusieurs déterminants importants des coûts d'exploitation :

- le volume d'eau vendu $(V)$;

- les prix des facteurs de production $(w)$;

- des caractéristiques techniques $(C A R)$ :

- le nombre d'abonnés desservis $(A b)$ et la longueur du réseau (Res), ou leur ratio (Dens $A b)$;

- le taux de rendement $(r d t)$ ou l'indice linéaire de pertes en distribution $(I L P)$;

- l'origine des eaux brutes $(E B)$;

- la topographie de la zone de distribution (Topo);

- le type de traitement de potabilisation (Trait).

La fonction de coût variable conditionnelle de court terme peut s'écrire de la façon suivante :

$$
C V(V, w ; C A R),
$$

\footnotetext{
${ }^{18}$ Voir parmi d'autres Crain et ZardKoohi, [1978]; Feigenbaum et Teeples, [1983]; Teeples et Glyer, [1987]; BHaTtacharyYa et al., [1995].
} 
où $C V$ représente les coûts variables (minimum). Nous avons choisi d'étudier une fonction de coût variable (de court terme) plutôt qu'une fonction de coût total (de long terme) pour plusieurs raisons. D'abord, une fonction de coût variable contient la même information que le processus de production d'origine. Ensuite, la période d'observation de notre échantillon est courte : quatre années pour les services en gestion déléguée et trois pour les services en régie. Par conséquent, une fonction de coût total faisant l'hypothèse que tous les facteurs (y compris le capital) peuvent s'ajuster instantanément serait une mauvaise spécification. Enfin, nous souhaitons minimiser l'impact de la différence de coûts du capital entre les contrats d'affermage (le mode de gestion déléguée présent exclusivement dans notre échantillon et largement dominant en France) et la gestion publique. Dans le cas d'affermage du service, les gros investissements et renouvellements sont en général à la charge de la collectivité et l'exploitant n'a qu'à effectuer les travaux de maintenance. Les dépenses de renouvellement varient fortement d'un contrat à l'autre suivant que le renouvellement à la charge du délégataire englobe seulement le matériel électromécanique ou davantage d'éléments du service. La responsabilité du renouvellement est partagée entre la collectivité et le délégataire, entraînant ainsi des transferts de charges financières. À l'opposé, les dépenses d'exploitation sont communes à l'ensemble des contrats de délégation mais aussi des comptes administratifs des régies même si l'agrégation des charges n'est pas la même.

\subsection{Description des données}

Nous n'utiliserons pas toutes les variables spécifiées dans la fonction de coût (1) pour différentes raisons. Certaines variables explicatives des coûts énoncés plus haut n'ont pas été introduites dans cette modélisation soit parce que les données n'étaient pas disponibles (c'est le cas pour la variable de topographie), soit parce que les observations ne présentaient que peu de variation pour avoir un effet significatif (eaux brutes, traitement). La liste des variables utilisées étant déjà assez longue, le préjudice n'en est que plus faible.

Nous avons à notre disposition deux bases de données. Il s'agit pour la première de services de distribution d'eau potable de la région de Bordeaux, délégués à des entreprises privées sous contrat d'affermage [Lyonnaise des eaux (groupe Suez), Générale des eaux (aujourd'hui Veolia eau), CISE, SAUR, Electricité Service Gironde (ESG) et SOGEDO $]^{19}$. Le second échantillon est exclusivement composé de services en régie localisés dans un département du nord de la France ${ }^{20}$.

L'échantillon des services en gestion déléguée comprend 188 observations concernant 47 services

\footnotetext{
${ }^{19}$ Depuis la date de collecte des données, il y a eu de nombreux changements dans l'organisation des sociétés distributrices d'eau potable. Cise est devenue filiale de SAUR en 1998 et le groupe Bouygues a cédé SAUR à PAI Partners fin 2004. ESG détenue en partie par Suez a été vendue à EDF en 2000 suite aux dégâts importants de la tempête de 1999.

${ }^{20}$ Pour des raisons de confidentialité, nous ne pouvons pas dévoiler l'origine exacte de ces données.
} 
communaux ou syndicaux sur les années 1995, 1996, 1997 et 1998. Nos données sont principalement issues des rapports réalisés par la Direction Départementale de l'Agriculture et de la Forêt (DDAF) de Gironde à partir des comptes-rendus techniques et financiers établis annuellement par les délégataires. Ces rapports contiennent des informations sur les dépenses en facteurs de production, des renseignements techniques sur le réseau, ainsi que des données sur les volumes d'eau, les consommations d'électricité etc. Une partie des données provient également d'une enquête que nous avons réalisée directement auprès des collectivités et du service commercial d'ESG qui nous a permis de consulter ses documents comptables et techniques. L'échantillon "gestion publique" comprend 52 services observés sur les années 1995, 1996 et 1997, soit 156 observations. Il s'agit d'informations provenant des comptes administratifs pour les données financières et des comptesrendus techniques pour les volumes d'eau et les données concernant la technologie et le réseau.

L'une des différences majeures avec l'échantillon sur les services en gestion déléguée est qu'il n'est pas possible de distinguer les dépenses et les quantités correspondant au facteur travail de celles ayant trait aux travaux de réparation et de sous-traitance. Nous avons donc décidé de regrouper les dépenses d'exploitation en trois catégories constantes quelque soit le mode de gestion et l'opérateur privé : dépenses d'électricité $(E)$, dépenses de traitement (pour potabiliser l'eau extraite) et d'analyse (pour contrôler la qualité de l'eau potable) $(T)$, et un ensemble de coûts (assez hétérogène) que nous avons nommé "autres dépenses" $(A)$. La somme de ces trois types de dépenses (variables) constitue les coûts d'exploitation $(C V)$, exprimés en euros, du service d'eau potable. Cette classification est intéressante du point de vue de la performance des services puisqu'elle nous permet d'isoler les dépenses qui peuvent être la source d'inefficacité dans une seule catégorie $(A)$. En effet, pour certains facteurs de production comme le travail, les travaux de réparation et de sous-traitance, le responsable du service n'est pas capable de dire si la quantité fournie est optimale pour produire les volumes d'eau demandés : il ne connaît pas l'effort que peut réaliser son personnel pour les tâches qui lui sont assignées et encore moins les capacités des entreprises auxquelles il confie la réalisation de certains travaux. Par ailleurs, nous pensons que ces regroupements de dépenses ne posent pas de problèmes économétriques importants puisque l'objet de l'étude n'est pas d'étudier les effets de substitution entre les facteurs de production ${ }^{21}$.

Le prix de l'électricité $\left(w_{E}\right)$ est défini comme le rapport entre les dépenses en électricité et la consommation annuelle d'énergie et a donc comme unité des $€ / k W h$. Comme il n'a pas été possible de trouver une mesure adéquate des quantités utilisées de facteurs donnant lieu à des dépenses de traitement et d'analyse, nous utilisons les quantités d'eau produites (et importées) afin de calculer un prix unitaire au $m^{3}\left(w_{T}\right)$ pour cette catégorie de facteurs. Par ailleurs, comme nous

\footnotetext{
${ }^{21}$ Il est aussi peu probable que cet arrangement soit la cause d'un biais lors de l'estimation puisque le facteur travail n'est pas une variable omise mais plutôt un élément que nous n'avons pas isolé. Par conséquent, le paramètre associé au prix du facteur $A$ devrait capter également les effets liés au facteur travail.
} 
l'avons indiqué plus haut, la catégorie $A$ de facteurs de production est composée de différents coûts assez hétérogènes regroupant des lignes comptables aussi diverses que achats et stocks, travaux et réparations, sous-traitance, et dépenses en salaires. En raison de l'absence d'informations sur les prix et du problème d'hétérogénéité de ce facteur, nous avons choisi de construire un indice de prix noté $w_{A}$ comme un coût unitaire par $m^{3}$ d'eau potable mis en distribution. L'hypothèse implicite dans la construction de ces prix est que les quantités physiques non observées des facteurs de production sont corrélées positivement avec les volumes d'eau.

Le volume d'eau produit qui a été effectivement comptabilisé $(V)$ est la somme du volume facturé aux usagers finals et du volume vendu à d'autres services d'eau potable. Ces quantités d'eau sont exprimées en $\mathrm{m}^{3}$. L'indice linéaire de pertes en distribution (ILP) est le volume des pertes $V_{p}$ (volume d'eau mis en distribution - volume d'eau comptabilisé) rapporté à la longueur des canalisations, et permet de comparer l'état physique de deux réseaux. Dans notre modèle de coût, nous utilisons également des variables techniques comme le nombre d'abonnés $(A b)$ et la longueur des canalisations (Res) exprimée en kilomètres, représentant la taille du réseau. Le ratio de ces deux variables $A b /$ Res donne la densité d'abonnés au km (appelé Dens $A b$ ). De même, on calcule la densité de production (Dens $P$ ) comme le volume distribué par abonné. Le nombre de communes constituant le service d'AEP (supérieur à 1 dans le cas d'un syndicat intercommunal) donne une idée des économies d'échelle pouvant être réalisées par une rationalisation de la gestion des finances et de l'aménagement du territoire.

Les statistiques descriptives des variables utilisées pour les deux types de gestion figurent dans le tableau 1. Les services en régie sont dans l'ensemble plus petits puisqu'ils desservent moins d'abonnés, distribuent moins de $m^{3}$ d'eau potable et présentent une longueur totale de canalisations moindre. La densité de production est un peu supérieure pour les services en gestion publique mais la densité d'abonnés est sensiblement la même pour les deux échantillons. Les valeurs moyennes de l'indice linéaire de pertes indiquent un état du réseau de distribution meilleur pour les services en gestion déléguée. Les services des deux échantillons tirent leurs eaux brutes de nappes souterraines et elles bénéficient des mêmes types de traitement pour le processus de potabilisation. La topographie est également similaire pour chaque région puisque dans les deux cas la configuration géographique est plutôt la plaine. Les infrastructures de mise en pression ne devraient pas par conséquent être très différentes. Enfin, les coûts variables moyens des services $(C V M)$ pour chaque échantillon sont en moyenne très proches $(0,62 €$ pour les services en régie et $0,58 €$ pour les services délégués). 
TAB. 1 - Statistiques descriptives des échantillons

\begin{tabular}{|c|c|c|c|c|c|c|c|c|c|c|c|}
\hline \multirow[b]{2}{*}{ Variable } & \multirow[b]{2}{*}{ Unité } & \multicolumn{5}{|c|}{$\begin{array}{l}\text { Gestion publique } \\
\text { (156 observations) }\end{array}$} & \multicolumn{5}{|c|}{$\begin{array}{l}\text { Gestion déléguée } \\
\text { (188 observations) }\end{array}$} \\
\hline & & Moy & Moyg & Std & Min & Max & Moy & Moyg & Std & Min & Max \\
\hline$C V$ & $\epsilon$ & 56250 & 34526 & 69030 & 4320 & 421078 & 212544 & 141278 & 255290 & 20513 & 1556402 \\
\hline$w_{E}$ & $€ / \mathrm{kwh}$ & 0,126 & 0,107 & 0,132 & 0,056 & 1,225 & 0,075 & 0,070 & 0,033 & 0,022 & 0,260 \\
\hline$w_{T}$ & $€ / m^{3}$ & 0,011 & 0,009 & 0,009 & 0,002 & 0,079 & 0,013 & 0,011 & 0,009 & 0,001 & 0,047 \\
\hline$w_{A}$ & $\epsilon / m^{3}$ & 0,377 & 0,353 & 0,142 & 0,096 & 1,098 & 0,379 & 0,366 & 0,107 & 0,184 & 0,860 \\
\hline$V$ & $m^{3} \quad 1$ & 105230 & 60183 & 148227 & 11046 & 869661 & 397276 & 252380 & 510368 & 31755 & 3177604 \\
\hline$V_{p}$ & $m^{3}$ & 52924 & 23095 & 93295 & 1613 & 713527 & 148766 & 81945 & 220597 & 4279 & 1258316 \\
\hline$I L P$ & $m^{3} / \mathrm{km}$ & 1601 & 1081 & 1699 & 81 & 12882 & 1081 & 869 & 788 & 182 & 4826 \\
\hline Res & $\mathrm{km}$ & 40 & 21 & 74 & 4 & 500 & 143 & 94 & 160 & 9 & 890 \\
\hline$A b$ & -- & 710 & 418 & 992 & 81 & 6028 & 2876 & 1898 & 3249 & 257 & 17210 \\
\hline Com & -- & 5 & 3 & 6 & 1 & 37 & 5 & 3 & 7 & 1 & 34 \\
\hline Dens $V$ & $m^{3} / \mathrm{Ab}$ & 209,1 & 201,0 & 63,7 & 117,4 & 574,4 & 180,2 & 177,2 & 33,6 & 107,9 & 304,8 \\
\hline Dens $A b$ & $\mathrm{Ab} / \mathrm{km}$ & 22,4 & 19,6 & 12,5 & 7,8 & 66,6 & 23,2 & 20,1 & 12,3 & 6,4 & 52,6 \\
\hline$C V M$ & $\epsilon$ & 0,616 & 0,573 & 0,223 & 0,133 & 1,405 & 0,579 & 0,560 & 0,156 & 0,259 & 1,218 \\
\hline
\end{tabular}

Notes : Moy $=$ moyenne arithmétique, Moyg $=$ moyenne géométrique, Std = écart-type.

\section{Modélisation économique du choix de mode de gestion}

L'objectif est d'étudier les interactions entre mode de gestion et coûts d'exploitation du service. La collectivité locale qui est responsable de l'organisation des services a deux options possibles : elle peut gérer elle-même le service ou bien en confier l'exploitation à une entreprise extérieure. Chaque mode de gestion engendre des coûts d'exploitation différents. On suppose que le choix de mode de gestion se fait à partir de la différence de coûts et des préférences spécifiques de chaque collectivité locale.

\subsection{Les coûts des services}

À partir de l'équation (1), on peut réécrire la fonction de coût variable pour le service $i$ et le mode de gestion $g$ en incorporant l'effet de variables aléatoires de la façon suivante :

$$
C V_{i}^{g} \equiv C V_{i}^{g}\left(R_{i}^{g}, \theta_{i}^{g}+\varepsilon_{c i}^{g}\right)
$$

où $C V_{i}^{g}$ représente les coûts variables du service $i$ pour le mode de gestion $g=0,1$, avec l'indice '0' pour la régie publique et l'indice '1' pour la gestion déléguée. $R_{i}^{g}$ est le vecteur des variables explicatives des coûts variables. Il comprend $V_{i}^{g}$ le volume d'eau vendu, $w_{i}^{g}$ le vecteur de prix des inputs et $C A R_{i}^{g}$ le vecteur des caractéristiques techniques du service d'eau potable. Toutes ces variables sont parfaitement observables par l'exploitant du service, la collectivité locale et l'économètre. Dans notre modèle, l'économètre dispose de la même information que la collectivité sauf pour la variable $\theta_{i}^{g}$ qui représente l'efficacité productive de l'exploitant. On considère que $\theta_{i}^{g}$ 
a deux composantes dont une seule est observable par la collectivité. Ainsi, $\theta_{i}^{g}$ s'écrit de la façon suivante :

$$
\theta_{i}^{g}=\theta_{i}^{o b^{g}}+\theta_{i}^{n o b^{g}}
$$

où $\theta_{i}^{\text {obg }}$ n'est observée que par la collectivité (et non par l'économètre). Dans le cas d'une gestion déléguée, la collectivité peut avoir une information sur la qualité de service de l'entreprise parce que cette dernière a déjà été en charge du service dans le passé ou bien parce qu'une commune voisine est en contrat avec cette entreprise. La composante $\theta_{i}^{n o b^{g}}$ n'est pas observable (ni par la collectivité, ni par l'économètre). Cette dernière reflète l'information privée de l'exploitant.

Le terme d'erreur $\varepsilon_{c i}^{g}$ capture l'existence d'aléas sur les coûts, non observés par la collectivité et l'exploitant. On note $C F_{i}$ les coûts fixes du service qui sont considérés comme exogènes et sont supposés ne pas dépendre du mode de gestion. Les coûts fixes sont majoritairement supportés par la collectivité locale quelque soit le mode gestion ${ }^{22}$. Pour cette raison, nous considérons que le choix de la collectivité se base uniquement sur les coûts variables.

\subsection{Le choix de mode de gestion}

La collectivité locale peut gérer elle-même le service d'eau (gestion directe publique, indice '0') ou bien confier son exploitation à une entreprise spécialisée (gestion déléguée, indice ' 1 '). Elle établit les coûts d'exploitation de son service en gestion directe. Dans le cas où elle souhaite déléguer le service, elle lance un appel d'offre stipulant essentiellement un niveau de service. C'est à partir des comptes d'exploitation prévisionnels que le maire choisit les candidats admis à négocier. Ces comptes sont élaborés dans la phase pré-contractuelle et présentent les dépenses auxquelles fait face le délégataire pour gérer le service et les recettes qu'il espère en tirer (CANNEVA et al., [2004]). On suppose que le choix du mode de gestion se fait à partir de la comparaison des coûts -la partie des coûts observée par la collectivité- pour un niveau de service donné. Nous définissons un modèle à régimes (switching model) à la LEE [1978] sur la base des fonctions de coût de chaque mode de gestion, et d'une fonction critère (ou équation de sélection) décrivant le choix ${ }^{23}$. La fonction critère est définie en supposant que le choix se fait à une période donnée précédant l'exploitation observée du service. La collectivité locale $i$ préfère la gestion déléguée à la gestion directe de son service d'eau si :

$$
E\left(C V_{i}^{0}\right)-E\left(C V_{i}^{1}\right)>\kappa_{i},
$$

\footnotetext{
${ }^{22}$ Dans certains cas d'affermage avec clauses concessives, une partie de ces coûts est transférée à l'opérateur chargé de l'exploitation du service. La partie fixe du tarif binôme est, en théorie, destinée à couvrir ces coûts fixes.

${ }^{23}$ À notre connaissance, seul le travail de HuANG et al. [2002] propose une application d'un modèle avec biais de sélection à la mesure d'efficacité de coût.
} 
où $E\left(C V_{i}^{0}\right)$ et $E\left(C V_{i}^{1}\right)$ sont les espérances des coûts variables conditionnellement aux observables de la collectivité, respectivement pour la gestion publique et la gestion déléguée. Cela signifie que la collectivité choisit la gestion déléguée si la différence de coûts est supérieure à une valeur de réserve non observée $\kappa_{i}$. Cette variable $\kappa_{i}$ peut être interprétée comme la prédisposition de la collectivité locale à déléguer son service d'eau potable, et peut ainsi être positive ou négative. On suppose que $\kappa_{i}$ est fonction de variables qui déterminent les coûts du service (consommation des usagers $V_{i}$ et caractéristiques du service $C A R_{i}$ ) mais aussi de variables qui n'affectent pas le coûts d'AEP comme le mode de gestion du service d'assainissement de cette même collectivité $i$, noté $A S S_{i}$ :

$$
\kappa_{i}=\alpha_{0}+\alpha_{V} V_{i}+\alpha_{C A R} C A R_{i}+\alpha_{A S S} A S S_{i}+\varepsilon_{i}
$$

où $\varepsilon_{i}$ est une erreur capturant des facteurs aléatoires non observables par la collectivité et l'exploitant, qui suit une loi $N\left(0, \sigma_{\varepsilon}^{2}\right)$. Ainsi, à partir des équations (4) et (5), on peut écrire l'équation structurelle de sélection de la façon suivante:

$$
I_{i}^{*}=\delta_{0}+\delta_{1}\left[E\left(C V_{i}^{0}\right)-E\left(C V_{i}^{1}\right)\right]+\delta_{V} V_{i}+\delta_{C A R} C A R_{i}+\delta_{A S S} A S S_{i}-\varepsilon_{i}
$$

En normalisant $\sigma_{\varepsilon}^{2}=1$, l'équation (6) a la forme d'un modèle Probit. Et donc si $I_{i}^{*}>0$, alors la collectivité locale $i$ choisit de déléguer l'exploitation de son service. En particulier, pour un différentiel de coûts $E\left(C V_{i}^{0}\right)-E\left(C V_{i}^{1}\right)$ positif, on s'attend à ce que $\delta_{1}$ soit positif.

\section{Analyse économétrique des coûts et du choix du mode de gestion}

Le modèle structurel à estimer est composé de deux équations de régime (fonctions de coût) et d'une équation de sélection. Nous utilisons des procédures d'estimation spécifiques aux données de panel (avec effets individuels corrélés) et correction du biais de sélection.

\subsection{Procédure d'estimation}

Nous disposons de données de panel. Nos variables sont alors indicées par $i$ représentant les individus et $t$ les périodes de temps. $i$ varie de 1 à $N_{0}+N_{1}$, avec $N_{0}$ le nombre de services en gestion directe et $N_{1}$ le nombre de services en gestion déléguée. Les service sont observés à partir de la période $t=1$. Le nombre de périodes total des services en gestion directe est notée $T_{0}\left(T_{1}\right.$ pour les services en gestion déléguée).

À partir de l'équation (2), nous réécrivons les équations de coût pour chaque régime (' 0 ' : gestion publique, '1' : gestion déléguée) dans lesquelles le vecteur des variables explicatives (maintenant indicé par $t) R_{i t}$ est composé de variables variant dans le temps $X_{i t}$ et de variables invariant 
dans le temps $Z_{i}$ :

$$
\begin{aligned}
& C V_{i t}^{0}=\beta^{0} X_{i t}^{0}+\gamma^{0} Z_{i}^{0}+\theta_{i}^{0}+\varepsilon_{c i t}^{0} \\
& C V_{i t}^{1}=\beta^{1} X_{i t}^{1}+\gamma^{1} Z_{i}^{1}+\theta_{i}^{1}+\varepsilon_{c i t}^{1},
\end{aligned}
$$

où $\varepsilon_{c i t}^{0}$ et $\varepsilon_{c i t}^{1}$ sont des termes d'erreur variant dans le temps suivant les distributions respectives $N\left(0, \sigma_{\varepsilon_{c}^{0}}^{2}\right)$ et $N\left(0, \sigma_{\varepsilon_{c}^{1}}^{2}\right)$. Les termes $\theta_{i}^{0}$ et $\theta_{i}^{1}$ sont des effets spécifiques aux individus (invariant dans le temps). Ils sont indépendants de $\varepsilon_{c i t}^{0}$ et $\varepsilon_{c i t}^{1}$. Ils représentent l'efficacité des exploitants et sont distribués aléatoirement de manière indépendante $N\left(0, \sigma_{\theta^{0}}^{2}\right)$ et $N\left(0, \sigma_{\theta^{1}}^{2}\right)$ respectivement ${ }^{24}$. Ils ne sont pas observés par l'économètre mais peuvent être reconstitués à partir des paramètres structurels estimés. Nous reviendrons sur ce point pour calculer l'efficacité des exploitants lors du choix de la spécification de la fonction de coût.

Le choix du mode de gestion intervient (de façon définitive) à la période 0 et se fait donc sur la base des coûts (et de ses déterminants) à cette période. À partir de la décomposition des effets individuels $\theta_{i}^{0}=\theta_{i}^{o b^{0}}+\theta_{i}^{n o b^{0}}$ et $\theta_{i}^{1}=\theta_{i}^{o 1^{1}}+\theta_{i}^{n o b^{1}}$, il est possible de réécrire l'équation structurelle de sélection (6) (qui ne varie pas dans le temps) sous une forme réduite :

$$
\begin{aligned}
I_{i}^{*} & =\delta_{0}+\delta_{1}\left[\beta^{0} X_{i 0}^{0}+\gamma^{0} Z_{i}^{0}+\theta_{i}^{o b^{0}}-\left(\beta^{1} X_{i 0}^{1}+\gamma^{1} Z_{i}^{1}+\theta_{i}^{o b^{1}}\right)\right] \\
& +\delta_{V} V_{i 0}+\delta_{C A R} C A R_{i 0}+\delta_{A S S} A S S_{i}-\varepsilon_{i} \\
& \equiv \lambda R_{i}+\delta_{A S S} A S S_{i}+u_{i},
\end{aligned}
$$

où $R_{i}$ est le vecteur des variables explicatives des coûts variables (incluant $V_{i 0}$ et $C A R_{i 0}$ ) observés à la période 0 . Le nouveau terme d'erreur $u_{i}$ s'écrit alors :

$$
u_{i}=\delta_{1}\left(\theta_{i}^{o b^{0}}-\theta_{i}^{o b^{1}}\right)-\varepsilon_{i}
$$

Le terme d'erreur $u_{i}$ dépend des effets individuels observés par la collectivité $\theta_{i}^{o b^{0}}$ et $\theta_{i}^{o b^{1}}$ et de $\varepsilon_{i}$, qui sont tous distribués selon une loi normale. $u_{i}$ suit donc aussi une loi normale de moyenne nulle et de variance $\sigma_{u}^{2}$. L'estimation des équations de coût doit prendre en compte le choix de la collectivité car cela introduit une corrélation entre le terme d'erreur $u_{i}$ et les $\theta_{i}^{o b}$ de chaque équation de coût. Il en résulte un biais de sélection.

Après normalisation de $\sigma_{u}^{2}=1, u_{i}$ suit une loi normale standard (centrée réduite) et l'équation (9) devient un modèle Probit. Si l'on note $\Psi=\lambda R_{i}+\delta_{A S S} A S S_{i}$, la règle de décision probabiliste

\footnotetext{
${ }^{24}$ Dans un modèle à effets fixes, les $\theta_{i}^{0}$ et $\theta_{i}^{1}$ sont considérés comme des constantes à estimer.
} 
du modèle s'écrit :

$$
\begin{aligned}
& \operatorname{Prob}\left(I_{i}=1\right)=\operatorname{Prob}\left(I_{i}^{*}>0\right)=\operatorname{Prob}\left(\Psi>-u_{i}\right)=\Phi(\Psi) \\
& \operatorname{Prob}\left(I_{i}=0\right)=\operatorname{Prob}\left(I_{i}^{*} \leq 0\right)=\operatorname{Prob}\left(\Psi \leq-u_{i}\right)=1-\Phi(\Psi)
\end{aligned}
$$

où $\Phi$ est la fonction de distribution cumulative de la loi normale standard ( $\phi$ fonction de densité).

En raison du biais de sélection, il faut ajuster les termes d'erreur des équations de coût de sorte que leur moyenne soit égale à 0 . Dans le cadre d'un modèle Probit, on peut écrire :

$$
\begin{aligned}
& E\left(\theta_{i}^{o b^{0}} \mid I_{i}=0\right)=\rho^{0} \frac{\phi(\Psi)}{1-\Phi(\Psi)} \\
& E\left(\theta_{i}^{o b^{1}} \mid I_{i}=1\right)=\rho^{1} \frac{-\phi(\Psi)}{\Phi(\Psi)}
\end{aligned}
$$

où $\frac{\phi(\Psi)}{\Phi(\Psi)}$ et $\frac{\phi(\Psi)}{1-\Phi(\Psi)}$ sont les ratios de Mills inverses pour chaque équation de régime. De plus, on a $\rho^{0}=\delta_{1} \operatorname{cov}\left(\theta_{i}^{o b^{0}}, u_{i}\right)$ et $\rho^{1}=\delta_{1} \operatorname{cov}\left(\theta_{i}^{o b^{1}}, u_{i}\right)$. On considère alors les équations de coût (conditionnellement à leur mode de gestion) suivantes:

$$
\begin{gathered}
C V_{i t}^{0}=\beta^{0} X_{i t}^{0}+\gamma^{0} Z_{i}^{0}+\rho^{0}\left(\frac{\phi(\Psi)}{1-\Phi(\Psi)}\right)+\eta_{i}^{0}+\varepsilon_{c i t}^{0} \\
C V_{i t}^{1}=\beta^{1} X_{i t}^{1}+\gamma^{1} Z_{i}^{1}+\rho^{1}\left(\frac{-\phi(\Psi)}{\Phi(\Psi)}\right)+\eta_{i}^{1}+\varepsilon_{c i t}^{1} .
\end{gathered}
$$

En incluant $\frac{-\phi(\Psi)}{\Phi(\Psi)}$ et $\frac{\phi(\Psi)}{1-\Phi(\Psi)}$ dans les équations de coût, on corrige le biais de sélection tel que $E\left(\eta_{i}^{0} \mid I_{i}=0\right)=0$ et $E\left(\eta_{i}^{1} \mid I_{i}=1\right)=0$, avec $\eta_{i}^{0}=\theta_{i}^{o b^{0}}+\theta_{i}^{n o b^{0}}-\rho^{0}\left(\frac{\phi(\Psi)}{1-\Phi(\Psi)}\right)$ et $\eta_{i}^{1}=\theta_{i}^{o b^{1}}+\theta_{i}^{n o b^{1}}-$ $\rho^{1}\left(\frac{-\phi(\Psi)}{\Phi(\Psi)}\right)$ les nouveaux effets individuels représentant l'efficacité de l'exploitant du service.

Nous utilisons une procédure d'estimation bien connue en deux étapes, due à HECKMAN [1979]. Les paramètres $\lambda$ et $\delta_{A S S}$ de l'équation de sélection réduite (9) -modèle Probit- sont d'abord estimés par la méthode du maximum de vraisemblance. Comme nous n'avons pas les informations sur les variables de coût (variant dans le temps) pour la période de signature de contrat, nous utilisons les valeurs disponibles à la période 1 . Les paramètres estimés $\hat{\lambda}$ et $\hat{\delta}_{A S S}$ nous permettent en outre de calculer les ratios de Mills inverses $\phi(\hat{\Psi}) / \Phi(\hat{\Psi})$ et $\phi(\hat{\Psi}) /(1-\Phi(\hat{\Psi}))$ avec $\hat{\Psi}=\hat{\lambda} R_{i}+$ $\hat{\delta}_{A S S} A S S_{i}$. Ces derniers sont ensuite reportés dans les équations de coût, et les paramètres $\beta$ et $\gamma$ sont estimés de manière convergente soit par la méthode de moindres carrés quasi-généralisés (MCQG), soit par des méthodes par variables instrumentales (VI) ou des méthodes Within selon les hypothèses d'exogénéité faites sur les variables explicatives. Dans notre modèle, les paramètres structurels des fonctions de coût sont identifiés grâce une condition d'exclusion basée sur la variable $A S S_{i}$ qui est un déterminant du choix de mode de gestion mais n'a pas d'impact sur les coûts, sans quoi l'identification serait due à la non-linéarité des ratios de Mills inverses. 
Pour chaque régime $j, \eta_{i}^{j}$ est i.i.d. $N\left(0, \sigma_{\eta^{j}}^{2}\right)$. Le terme d'erreur $\varepsilon_{c i t}^{j}$ est i.i.d. $N\left(0, \sigma_{\varepsilon_{c}^{j}}^{2}\right)$, et le terme d'erreur global s'écrit $v_{i t}^{j}=\eta_{i}^{j}+\varepsilon_{i t}^{j}$. On se place donc dans le cadre d'un modèle à effet individuel aléatoire et on a l'hypothèse suivante : $E\left(v^{j} v^{j^{\prime}}\right)=\Omega_{j}=t^{j} \sigma_{\eta^{j}}^{2} B+\sigma_{\varepsilon_{c}^{j}}^{2}(Q+B)$, où $B$ et $Q$ sont respectivement les matrices de transformation Between et Within. On corrige l'hétéroscédasticité présente dans le modèle à erreurs composées en diagonalisant la matrice $\Omega_{j}$, en utilisant les transformation suivantes :

$$
\begin{aligned}
& \widetilde{C V}^{j}=\sigma_{\varepsilon_{c}^{j}} \Omega_{j}^{-1 / 2} C V^{j}=\left(Q+\frac{\sigma_{\varepsilon_{c}^{j}}}{\bar{\sigma}^{j}} B\right) C V^{j} \\
& \widetilde{X}^{j}=\sigma_{\varepsilon_{c}^{j}} \Omega_{j}^{-1 / 2} X^{j}=\left(Q+\frac{\sigma_{\varepsilon_{c}^{j}}}{\bar{\sigma}^{j}} B\right) X^{j} \\
& \widetilde{Z}^{j}=\sigma_{\varepsilon_{c}^{j}} \Omega_{j}^{-1 / 2} Z^{j}=\left(Q+\frac{\sigma_{\varepsilon_{c}^{j}}}{\bar{\sigma}^{j}} B\right) Z^{j},
\end{aligned}
$$

où $\bar{\sigma}^{j 2}=\sigma_{\eta^{j}}^{2}+\frac{\sigma_{\varepsilon_{c}^{j}}^{2}}{t^{j}}$. On définit $\widetilde{H}^{j}=\left[\widetilde{X}^{j}, \widetilde{Z}^{j}, M i l l^{j}\right]$ où $M i l l^{0}=\frac{\phi}{1-\Phi}$ et $M i l l^{1}=\frac{-\phi}{\Phi}$, et $\Gamma^{j}=$ $\left[\beta^{j}, \gamma^{j}, \rho^{j}\right]$. Dans ce modèle à effets aléatoires, l'estimateur MCQG s'écrit :

$$
\hat{\Gamma}_{M C Q G}^{j} \equiv\left[\hat{\beta}_{M C Q G}^{j}, \hat{\gamma}_{M C Q G}^{j}, \hat{\rho}_{M C Q G}^{j}\right]=\left(\widetilde{H}^{j^{\prime}} \widetilde{H}^{j}\right)^{-1} \widetilde{H}^{j^{\prime}} \widetilde{C V}^{j}
$$

où les variances théoriques des erreurs sont remplacées par leur équivalent estimé (voir l'annexe A).

Dans un modèle à effets fixes, les $\eta_{i}^{j}$ sont considérés comme des constantes à estimer. La procédure de transformation Within faisant disparaître les effets fixes, cela implique que l'estimation n'est pas affectée par le biais de sélection. Pour la même raison, il n'est pas possible d'identifier les paramètres associés aux variables invariant dans le temps. Ainsi, seul $\beta^{j}$ est estimé :

$$
\hat{\beta}_{Q}^{j}=\left(X^{j^{\prime}} Q X^{j}\right)^{-1} X^{j^{\prime}} Q C V^{j}
$$

HAUSMAN [1978] propose un test d'exogénéité consistant à comparer l'estimateur Within à celui des MCQG ${ }^{25}$. L'hypothèse nulle $H_{0}$ testée dans le modèle de régression avec des données de panel est la non corrélation des variables $X^{j}$ et de l'effet individuel $\eta_{i}^{j}$. La statistique de test est donnée par : TH $=\hat{q}^{\prime}[\operatorname{var}(\hat{q})]^{-1} \hat{q}$, avec $\hat{q}=\hat{\beta}_{Q}^{j}-\hat{\beta}_{M C Q G}^{j}$. Sous l'hypothèse $H_{0}, T H$ est distribuée asymptotiquement selon un $\chi^{2}$ avec comme degrés de liberté le nombre de variables $X$. Si l'hypothèse $H_{0}$ est rejetée, alors on préférera utiliser un estimateur VI.

Si l'on indice par ' 1 ' les variables supposées exogènes et '2' les variables supposées endogènes, on pourra utiliser la matrice des instruments proposée par HAUSMAN et TAYLOR [1981], HT

\footnotetext{
${ }^{25} \mathrm{Si}$ l'hypothèse de non corrélation des effets individuels n'est pas rejetée par le test d'Hausman Within vs. MCQG mais que la régression contient des variables invariant dans le temps potentiellement corrélées avec les effets individuels, alors on préfère utiliser un test d'Hausman basé sur la comparaison des estimateurs VI et MCQG.
} 
ci-après :

$$
A_{H T}=\left[Q X, X_{1}, Z_{1}\right]
$$

Il existe des instruments supplémentaires qui améliorent l'efficacité de l'estimateur VI. AMEMIYA et MACURDY [1986], AM ci-après, proposent la matrice d'instruments suivante : $A_{A M}=$ $\left[Q X, X_{1 s}, Z_{1}\right]$ et Breusch et al. [1989], BMS ci-après, la matrice $A_{B M S}=\left[Q X, Q X_{1 s}, Q X_{2 s}, B X_{1}, Z_{1}\right]$ (voir Breusch et al. [1989], pour la définition des matrice $X_{1 s}$ et $X_{2 s}$ ). L'expression générale de l'estimateur VI est :

$$
\hat{\Gamma}_{V I}^{j}=\left(\bar{H}^{j^{\prime}} P_{A}^{j} \bar{H}^{j}\right)^{-1} \bar{H}^{j^{\prime}} P_{A}^{j} \overline{C V^{j}},
$$

avec $P_{A}^{j}=A^{j}\left(A^{j^{\prime}} A^{j}\right)^{-1} A^{j^{\prime}}$ où $A^{j}$ est la matrice des instruments. $\bar{H}$ et $\overline{C V}$ sont définis de la même façon que $\widetilde{H}$ et $\widetilde{C V}$ mais prennent aussi en compte l'endogénéité de certaines variables, voir HaUsman et TAYLOR [1981]. On utilise un test d'Hausman pour vérifier la validité des instruments additionnels. La statistique de test suit une loi $\chi^{2}$ avec comme degrés de liberté le nombre d'instruments supplémentaires.

Dans l'estimation en deux étapes, si l'on ignore que les ratios de Mills inverses introduits comme variables explicatives dans le seconde étape sont construits à partir des coefficients estimés en première étape, alors la matrice de variance-covariance des paramètres estimés dans la seconde étape est sous-estimée. Les matrices de variance-covariance sont donc corrigées comme dans LEE et al. [1980] en tenant compte de la structure panel des échantillons. Ces matrices pour les estimateurs MCQG, Within et VI sont détaillées dans l'annexe $\mathrm{A}^{26}$.

Enfin, les paramètres de l'équation structurelle Probit (6) peuvent être estimés par une méthode de maximum de vraisemblance sur les variables explicatives $V_{i}, C A R_{i}, A S S_{i}$ et le différentiel de coût (estimé) $\widehat{C V}_{i}^{0}-\widehat{C V}_{i}^{1}$. Lorsque les coûts sont effectivement observés, les coûts estimés sont calculés à partir des espérances conditionnelles :

$$
\begin{aligned}
& E\left(C V_{i t}^{0} \mid I=0\right)=\beta^{0} X_{i t}^{0}+\gamma^{0} Z_{i}^{0}+\rho^{0}\left(\frac{\phi(\hat{\Psi})}{1-\Phi(\hat{\Psi})}\right) \\
& E\left(C V_{i t}^{1} \mid I=1\right)=\beta^{1} X_{i t}^{1}+\gamma^{1} Z_{i}^{1}+\rho^{1}\left(\frac{-\phi(\hat{\Psi})}{\Phi(\hat{\Psi})}\right)
\end{aligned}
$$

Lorsque les coûts ne sont pas observés parce que la collectivité n'a pas fait le choix du mode de gestion alternatif, les coûts sont estimés à partir du calcul de l'espérance des coûts conditionnellement

\footnotetext{
${ }^{26}$ Dans le modèle à effets fixes, les ratios de Mills inverses n’apparaissent pas dans la régression car éliminés par la transformation Within, la matrice de variance-covariance n'a donc pas besoin d'être corrigée.
} 
au régime qui aurait pu être choisi :

$$
\begin{aligned}
& E\left(C V_{i t}^{0} \mid I=1\right)=\beta^{1} X_{i t}^{0}+\gamma^{1} Z_{i}^{0}+\rho^{1}\left(\frac{-\phi(\hat{\Psi})}{\Phi(\hat{\Psi})}\right) \\
& E\left(C V_{i t}^{1} \mid I=0\right)=\beta^{0} X_{i t}^{1}+\gamma^{0} Z_{i}^{1}+\rho^{0}\left(\frac{\phi(\hat{\Psi})}{1-\Phi(\hat{\Psi})}\right) .
\end{aligned}
$$

\subsection{Application empirique}

\subsubsection{Spécification de la fonction de coût}

La fonction de coût variable est spécifiée selon la forme flexible translog (CHRISTENSEN et al., [1971]). L'avantage est qu'elle impose peu de restrictions a priori sur les caractéristiques de la technologie. C'est une approximation locale, nous choisissons la moyenne des variables (en $\log$ ) comme point de référence ${ }^{27}$. Ainsi, les coefficients de premier ordre peuvent être interprétés directement comme des élasticités de coût évalués à la moyenne (géométrique) de l'échantillon. La fonction de coût variable translog s'écrit :

$$
\begin{aligned}
\ln (C V) & =a_{0}+\sum_{h} a_{h} \ln w_{h}+a_{v} \ln V+\sum_{k} b_{k} \ln C A R_{k} \\
& +\frac{1}{2} \sum_{h} \sum_{h^{\prime}} a_{h h^{\prime}} \ln w_{h} \ln w_{h^{\prime}}+\frac{1}{2} b_{v v}(\ln V)^{2}+\sum_{h} b_{h v} \ln w_{h} \ln V,
\end{aligned}
$$

où $C V$ représente les coûts variables, $w_{h}$ le prix de l'input $h, V$ le volume d'eau vendu et $C A R_{k}$ la variable technique $k$. L'ensemble des caractéristiques des services retenues est : $C A R=$ $\{I L P, A b, C o m\}$. Les paramètres à estimer sont : $\left(a_{0}, a_{h}, a_{v}, b_{k}, a_{h h^{\prime}}, b_{v v}, b_{h v}\right)$. Nous supposons que la fonction de coût satisfait les restrictions de symétrie suivantes : $a_{h h^{\prime}}=a_{h^{\prime} h}$. En outre, une fonction de coût bien définie doit respecter la propriété d'homogénéité de degré 1 par rapport aux prix des facteurs. Cette propriété ${ }^{28}$ est imposée avant l'estimation de la fonction de coût en divisant les coûts variables et les prix des facteurs de production par le prix d'un facteur référent, ici le prix du facteur $A$ (catégorie d'autres facteurs que travail et électricité). Plutôt que d'imposer d'autres restrictions sur la forme globale de la fonction de coût qui pourraient restreindre les propriétés de flexibilité de la forme translog, nous nous assurons de sa concavité, une fois les paramètres de la fonction de coût estimés, en vérifiant que la matrice des coefficients $\left\{a_{h h^{\prime}}\right\}$ est bien semi-définie négative (DIEWERT et WALES, [1987]).

Nous réalisons plusieurs tests pour vérifier certaines propriétés de la technologie. Le test d'élas-

\footnotetext{
${ }^{27} \mathrm{La}$ fonction de coût est estimée par rapport à la moyenne des variables explicatives c'est-à-dire le logarithme des variables observées $: \ln X$. Ainsi, toutes les variables à droite de l'équation sont normalisées par leur moyenne géométrique sur l'échantillon en retranchant $E(\ln X)$.

${ }^{28}$ Il est équivalent d'imposer un ensemble de contraintes sur les paramètres de la fonction de coût : $\sum_{h} a_{h}=$ $1, \sum_{h} a_{h h^{\prime}}=\sum_{h^{\prime}} a_{h h^{\prime}}=0, \sum_{h} b_{h v}=0$.
} 
ticité de substitution unitaire consistant à imposer les restrictions paramétriques $a_{h h^{\prime}}=0, \forall h, h^{\prime}$, a pour objectif de valider ou non la séparabilité des facteurs de production. Nous testons aussi l'hypothèse d'homothétie de la technologie (indépendance des prix des facteurs et des niveaux de production). Cela revient à imposer que les paramètres correspondant aux produits croisés entre prix des facteurs et quantités produites sont conjointement égaux à zéro : $b_{h v}=0, \forall h$. Enfin, nous testons si la technologie ne se réduit pas à une fonction de coût Cobb-Douglas. Pour cela, il suffit de tester la significativité des paramètres correspondant aux termes quadratiques de la forme translog, ce qui revient à imposer les restrictions sur les paramètres suivantes : $a_{h h^{\prime}}=0, b_{v v}=0, b_{h v}=0, \forall h, h^{\prime}$.

\subsubsection{Résultats d'estimation}

Nous présentons dans le tableau 5 (colonne 1) les estimations des paramètres du modèle Probit (9) représentant l'équation de sélection sous sa forme réduite. Les paramètres estimés permettent de calculer les ratios de Mills inverses qui servent à corriger un possible biais de sélection dans l'estimation des fonctions de coût variable. Rappelons que l'ensemble des déterminants du choix de mode gestion (sous sa forme réduite) est composé des variables explicatives des coûts, notées $R$, mais aussi de la variable $A S S$ (qui n'affecte pas les coûts et qui sert de condition d'exclusion). Les fonctions de coût sont estimées avec les différentes méthodes vues ci-dessus; les résultats sont présentés dans le tableau 2.

Pour l'échantillon de services en régie, le test d'Hausman consistant à comparer les estimateurs Within et MCQG ne rejette pas l'hypothèse nulle de non corrélation des variables explicatives et de l'effet individuel. Nous avons aussi calculé la statistique de test comparant les estimateurs VI-HT et MCQG dont la valeur est 16,77 et la p-value 0,16 conduisant au même résultat. L'estimateur des MCQG est donc le meilleur estimateur sans biais. En revanche, concernant la fonction de coût en gestion déléguée, la valeur de la statistique de test est égale à 281,26 sous l'hypothèse nulle $H_{0}$ de non corrélation. On rejette donc l'hypothèse $H_{0}$ avec un risque bien inférieur à $1 \%$ et on utilise une méthode par VI. Dans ce cas, il est nécessaire de faire des hypothèses d'exogénéité. Nous considérons que les volumes d'eau produits ne sont pas exogènes car, comme le montrent GARCIA et Thomas [2003], ce sont des variables de décision des exploitants des services qui entrent dans le contrat de délégation et dépendent du paramètre $\theta$ d'efficacité. Ainsi, ni la variable $(\ln V$ ) (et son terme au carré $\left.(\ln V)^{2}\right)$ ni la variable $(\ln I L P)$ (qui est construite à partir du volume d'eau perdue $V_{p}$ ) ne sont présents dans la matrice des instruments $A_{H T}$ donnée par la formule (13). Le résultat du test d'Hausman (avec une valeur de 6,66) valide nos hypothèses (p-value égale à 0,35). Comme expliqué plus haut, on peut utiliser des instruments supplémentaires pour accroître 
TAB. 2 - Estimations des fonctions de coût variable

\begin{tabular}{|c|c|c|c|c|c|}
\hline \multirow[b]{2}{*}{ Variable } & \multicolumn{2}{|c|}{ Gestion publique } & \multicolumn{3}{|c|}{ Gestion déléguée } \\
\hline & Within & MCQG & Within & MCQG & BMS \\
\hline Constante & & $\begin{array}{l}12,1003^{* * *} \\
(0,0252)\end{array}$ & & $\begin{array}{l}12,2563^{* * *} \\
(0,0094)\end{array}$ & $\begin{array}{l}12,2967^{* * *} \\
(0,0437)\end{array}$ \\
\hline $\ln V$ & $\begin{array}{c}0,4003^{* * *} \\
(0,1167)\end{array}$ & $\begin{array}{c}0,3456^{* * *} \\
(0,0368)\end{array}$ & $\begin{array}{c}0,4951^{* * *} \\
(0,0308)\end{array}$ & $\begin{array}{c}0,5706^{* * *} \\
(0,0216)\end{array}$ & $\begin{array}{c}0,5091 \text { *** } \\
(0,0296)\end{array}$ \\
\hline $\ln w_{E}$ & $\begin{array}{c}0,1467 \\
(0,0388)\end{array}$ & $\begin{array}{c}0,1937^{* * *} \\
(0,0247)\end{array}$ & $\begin{array}{c}0,0797^{* * *} \\
(0,0082)\end{array}$ & $\begin{array}{c}0,0937^{* * *} \\
(0,0073)\end{array}$ & $\begin{array}{l}0,0823^{* * *} \\
(0,0082)\end{array}$ \\
\hline $\ln w_{T}$ & $\begin{array}{r}-0,0058 \\
(0,0213)\end{array}$ & $\begin{array}{r}-0,0125 \\
(0,0156)\end{array}$ & $\begin{array}{c}0,0328^{* * *} \\
(0,0083)\end{array}$ & $\begin{array}{c}0,0498^{* * *} \\
(0,0069)\end{array}$ & $\begin{array}{l}0,0379^{* * *} \\
(0,0081)\end{array}$ \\
\hline $\ln I L P$ & $\begin{array}{c}0,2316^{* * *} \\
(0,0210)\end{array}$ & $\begin{array}{c}0,1993^{* * *} \\
(0,0114)\end{array}$ & $\begin{array}{c}0,1995^{* * *} \\
(0,0102)\end{array}$ & $\begin{array}{c}0,1612 \\
(0,0066)\end{array}$ & $\begin{array}{l}0,2015^{* * *} \\
(0,0096)\end{array}$ \\
\hline $\ln A b o n$ & $\begin{array}{r}0,7220 \\
(0,6020)\end{array}$ & $\begin{array}{c}0,4640 \\
(0,0347)\end{array}$ & $\begin{array}{c}0,2480 \\
(0,0843)\end{array}$ & $\begin{array}{c}0,3730^{* * *} \\
(0,0226)\end{array}$ & $\begin{array}{c}0,3832^{* * *} \\
(0,0448)\end{array}$ \\
\hline$(\ln V)^{2}$ & $\begin{array}{r}-0,0168 \\
(0,1074)\end{array}$ & $\begin{array}{r}-0,0458 \\
(0,0198)\end{array}$ & $\begin{array}{r}-0,0336 \\
(0,0307)\end{array}$ & $\begin{array}{r}-0,0179 \\
(0,0079)\end{array}$ & $\begin{array}{r}-0,0182 \\
(0,0244)\end{array}$ \\
\hline$\left(\ln w_{E}\right)^{2}$ & $\begin{array}{r}-0,0195 \\
(0,0440)\end{array}$ & $\begin{array}{c}-0,0914^{* * *} \\
(0,0277)\end{array}$ & $\begin{array}{c}0,0545^{* * *} \\
(0,0181)\end{array}$ & $\begin{array}{r}0,0015 \\
(0,0171)\end{array}$ & $\begin{array}{l}0,0535^{* * *} \\
(0,0180)\end{array}$ \\
\hline$\left(\ln w_{T}\right)^{2}$ & $\begin{array}{r}0,0361 \\
(0,0302)\end{array}$ & $\begin{array}{r}0,0494 \\
(0,0240)\end{array}$ & $\begin{array}{r}0,0227 \\
(0,0122)\end{array}$ & $\begin{array}{c}0,0487^{* * *} \\
(0,0108)\end{array}$ & $\begin{array}{r}0,02955^{* *} \\
(0,0121)\end{array}$ \\
\hline $\ln w_{E} \times \ln w_{T}$ & $\begin{array}{r}0,0493 \\
(0,0289)\end{array}$ & $\begin{array}{r}0,0526 \\
(0,0205)\end{array}$ & $\begin{array}{r}0,0137 \\
(0,0128)\end{array}$ & $\begin{array}{r}0,0233 \\
(0,0117)\end{array}$ & $\begin{array}{r}0,0145 \\
(0,0127)\end{array}$ \\
\hline $\ln$ Com & & $\begin{array}{c}0,2114 \\
(0,0211)\end{array}$ & & $\begin{array}{c}0,1032^{* * *} \\
(0,0061)\end{array}$ & $\begin{array}{l}0,1305^{* * *} \\
(0,0313)\end{array}$ \\
\hline Mill & & $\begin{array}{c}0,0885^{* * *} \\
(0,0195)\end{array}$ & & $\begin{array}{r}-0,0135 \\
(0,0082)\end{array}$ & $\begin{array}{r}0,0033 \\
(0,0100)\end{array}$ \\
\hline$R^{2}$ ajusté & 0,9382 & 0,9788 & 0,9036 & 0,9896 & 0,9852 \\
\hline
\end{tabular}

Notes : $N_{0} \times T_{0}=52 \times 3$ et $N_{1} \times T_{1}=47 \times 4$. Écarts-types entre parenthèses.

${ }^{*},{ }^{* *}$ et ${ }^{* * *}$ pour les niveaux de significativité respectifs de $10 \%, 5 \%$ et $1 \%$.

l'efficacité de l'estimateur VI. Les hypothèses de validité des matrices $A_{A M}$ et $A_{B M S}$ ne sont pas rejetées, nous concluons donc que l'estimateur BMS est le meilleur estimateur pour la fonction de coût de la gestion déléguée. Les résultats de ces tests sont présentés dans le tableau 3.

Les coefficients estimés de la variable de sélection appelée Mill sont reportés dans le tableau 2. Cette variable est calculée à partir de la formule $\phi(\hat{\Psi}) /(1-\Phi(\hat{\Psi}))$ pour la fonction de coût en gestion publique et $-\phi(\hat{\Psi}) / \Phi(\hat{\Psi})$ pour celle en gestion déléguée. Ce coefficient est significativement différent de zéro (à un niveau de 1\%) pour l'échantillon de régies mais pas pour l'échantillon de services délégués. Cela prouve l'existence d'un biais de sélection lorsque le choix de la collectivité n'est pas pris en compte dans l'estimation de la fonction de coût. Le signe positif du biais de sélection pour la gestion publique signifie que les coûts observés sont plus élevés que ce qu'ils auraient été si la collectivité avait choisi la gestion déléguée. En revanche, il n'y a pas de différence significative pour l'échantillon en gestion déléguée.

Les coefficients estimés des variables peuvent être directement utilisés pour le calcul des élasticités de coût du service moyen de chaque échantillon ${ }^{29}$ et l'estimation des coûts marginaux.

\footnotetext{
${ }^{29}$ Grâce à la normalisation des données (approximation de la fonction de coût autour de la moyenne), les para-
} 
TAB. 3 - Résultats des tests d'Hausman

\begin{tabular}{lcr}
\hline $\begin{array}{l}\text { Test } \\
\text { d'Hausman }\end{array}$ & $\begin{array}{c}\text { Gestion } \\
\text { publique }\end{array}$ & \multicolumn{1}{c}{$\begin{array}{c}\text { Gestion } \\
\text { déléguée }\end{array}$} \\
\hline Within vs. MCQG & $12,01(0,2125)$ & $194,93(0,0000)$ \\
VI-HT vs. MCQG & $16,77(0,1586)$ & $281,26(0,0000)$ \\
Within vs. VI-HT & & $6,66(0,3532)$ \\
VI-AM vs. VI-HT & & $2,32(0,9987)$ \\
VI-BMS vs. VI-AM & & $2,56(0,9998)$ \\
\hline
\end{tabular}

Notes : P-value entre parenthèses.

L'estimation des coûts variables totaux pour chaque mode de gestion et la prédiction des coûts variables totaux si l'autre mode de gestion avait été choisi comme décrit en fin de section 4.1 nous permet de calculer le différentiel de coûts entre les deux modes de gestion. Le coût marginal est calculé comme le produit du coût moyen et de l'élasticité du coût par rapport au volume $V$. Par exemple, en reprenant la spécification translog (14), le coût marginal en gestion publique est calculé de la façon suivante :

$$
\widehat{C m}_{i t}^{0}=\frac{\widehat{C V}^{0}}{V^{0}} \times\left(\widehat{a_{v}}+\widehat{b_{v v}} \ln V^{0}\right)
$$

où $\widehat{C V}^{0}=\exp \left(\widehat{a_{0}}+\sum_{h} \widehat{a_{h}} \ln w_{h}+\widehat{a_{v}} \ln V^{0}+\sum_{k} \widehat{b_{k}} \ln C A R_{k}^{0}+\frac{1}{2} \sum_{h} \sum_{h^{\prime}} \widehat{a_{h h^{\prime}}} \ln w_{h}^{0} \ln w_{h^{\prime}}^{0}+\frac{1}{2} \widehat{b_{v v}}\left(\ln V^{0}\right)^{2}+\right.$ $\left.\sum_{h} \widehat{b_{h v}} \ln w_{h}^{0} \ln V^{0}\right)$. On présente les statistiques descriptives des coûts marginaux estimés pour les deux sous-échantillons dans le tableau 4. On note que les coûts marginaux sont en moyenne plus bas pour le services en gestion publique car les services de ce sous-échantillon sont de plus petite taille que ceux en délégation.

TAB. 4 - Estimation des coûts marginaux

\begin{tabular}{lcc}
\hline & $\begin{array}{c}\text { Gestion } \\
\text { publique } \\
\text { (156 observations) }\end{array}$ & $\begin{array}{c}\text { Gestion } \\
\text { déléguée } \\
\text { (188 observations) }\end{array}$ \\
\hline Moyenne & 0,2359 & 0,2944 \\
Médiane & 0,2198 & 0,2773 \\
Std & 0,1036 & 0,1045 \\
Min & 0,0769 & 0,1249 \\
Max & 0,6354 & 0,8621 \\
\hline
\end{tabular}

L'équation structurelle (6) de choix de la collectivité qui dépend de ce différentiel et des caractéristiques du service peut ainsi être estimée. Les résultats sont reportés dans le tableau mètres de premier ordre peuvent être interprétés directement comme des élasticités de coût (estimées à la moyenne géométrique pour toutes les variables de coût de chaque échantillon). 
TAB. 5 - Estimation de l'équation de sélection

\begin{tabular}{|c|c|c|c|}
\hline Variable & $\begin{array}{l}\text { Forme } \\
\text { réduite }\end{array}$ & \multicolumn{2}{|c|}{$\begin{array}{l}\text { Forme } \\
\text { structurelle }\end{array}$} \\
\hline Constante & $\begin{array}{l}-0,5106 \\
(0,8967)\end{array}$ & $\begin{array}{r}0,1779 \\
(0,4974)\end{array}$ & \\
\hline $\ln V$ & $\begin{array}{r}-0,2962 \\
(4,0769)\end{array}$ & & \\
\hline $\ln w_{E}$ & $\begin{array}{c}-2,6621 \\
(1,5362)\end{array}$ & & \\
\hline $\ln w_{T}$ & $\begin{array}{l}2,3482 \\
(1,1502)\end{array}$ & & \\
\hline $\ln I L P$ & $\begin{array}{l}-1,2362 \\
(0,6685)\end{array}$ & $\begin{array}{c}-0,7639 \\
(0,3089)\end{array}$ & $* *$ \\
\hline $\ln A b$ & $\begin{array}{r}3,8569 \\
(4,4004)\end{array}$ * & $\begin{array}{r}1,8826 \\
(0,4645)\end{array}$ & $* * *$ \\
\hline$(\ln V)^{2}$ & $\begin{array}{l}-1,5790 \\
(0,8681)\end{array}$ & & \\
\hline$\left(\ln w_{E}\right)^{2}$ & $\begin{array}{l}-4,5942 \\
(4,2794)\end{array}$ & & \\
\hline$\left(\ln w_{T}\right)^{2}$ & $\begin{array}{r}2,4911 \\
(1,8665)\end{array}$ & & \\
\hline $\ln w_{E} \times \ln w_{T}$ & $\begin{array}{r}2,1151 \\
(2,6477)\end{array}$ & & \\
\hline $\ln C o m$ & $\begin{array}{l}-1,6404^{* *} \\
(0,6577)\end{array}$ & $\begin{array}{r}-1,6390 \\
(0,4657)\end{array}$ & $* * *$ \\
\hline$A S S$ & $\begin{array}{l}2,9932 \\
(1,3951)\end{array}$ & $\begin{array}{r}1,1838 \\
(0,5256)\end{array}$ & $* *$ \\
\hline$\widehat{\ln C^{0}}-\widehat{\ln C^{1}}$ & & $\begin{array}{r}5,5160 \\
(2,5547) \\
\end{array}$ & $* *$ \\
\hline $\begin{array}{l}\text { Pseudo } R^{2} \text { McFadden } \\
\text { Bonnes prédictions }\end{array}$ & $\begin{array}{r}0,8597 \\
97 \%\end{array}$ & $\begin{array}{r}0,7409 \\
94 \%\end{array}$ & \\
\hline
\end{tabular}

Notes : $N_{0}+N_{1}=99$. Écarts-types entre parenthèses.

${ }^{*},{ }^{* *}$ et ${ }^{* * *}$ pour les niveaux de significativité respectifs de $10 \%, 5 \%$ et $1 \%$.

5 (colonne 2). La forme structurelle du choix de mode de gestion est estimée de manière très satisfaisante avec un pseudo $R^{2}$ égal à 0,74 et $94 \%$ des observations correctement prédites. Les variables explicatives ont toutes un impact significatif sur le choix de mode de gestion ${ }^{30}$. Le paramètre relatif au différentiel de coûts est significativement positif à un niveau de $5 \%$, ce qui confirme que la collectivité choisira de déléguer son service d'eau si le coût associé à ce mode de gestion est inférieur à celui en régie. Un réseau de distribution dont l'état physique (mesuré par l'indice linéaire de pertes) est moins bon a une probabilité plus élevée de rester en régie. Toutes choses égales par ailleurs, une nombre d'usagers plus important augmente la probabilité d'une gestion déléguée du service d'eau. En revanche, un service composé d'un regroupement

\footnotetext{
${ }^{30}$ Initialement, le volume d'eau faisait partie des déterminants du choix de gestion mais il n'apparait pas comme une variable influant le choix, nous l'avons donc retiré du modèle Probit.
} 
de plusieurs communes a une probabilité plus faible d'être géré par une entreprise privée. En effet, plus le nombre de communes augmente, plus il est difficile d'obtenir un consensus pour la délégation. Enfin, si le service d'assainissement est géré par délégation, alors le service d'AEP a une grande chance d'être aussi délégué.

\subsubsection{Rendements et performance des services}

L'étude de la flexibilité des coûts variables en fonction du volume d'eau et d'autres variables déterminantes comme le nombre d'usagers donne des indications importantes sur la performance des services. Notre spécification des coûts variables nous permet de faire la distinction entre rendements de densité et rendements d'échelle. Nous pouvons ainsi étudier les différentes manières dont la production peut s'accroitre pour des activités en réseau et leur impact sur les coûts variables moyens.

Notons $V$ le volume d'eau facturé et $V_{p}$ le volume d'eau perdu. On parle d'économies de densité de production lorsque les coûts variables moyens diminuent lors d'une augmentation du volume d'eau produit $\left(V+V_{p}\right)$, mais à nombre d'abonnés $(A b)$ constant (la quantité produite par usager croît). Les rendements de densité de production sont mesurés par l'élasticité suivante : $\left[\frac{\partial \ln C V(w, V, C A R)}{\partial \ln V}+\frac{\partial \ln C V(w, V, C A R)}{\partial \ln V_{p}}\right]^{-1}$.

Par ailleurs, l'élasticité de densité d'usagers mesure les économies de coût réalisées lors de l'accroissement proportionnel de la production et du nombre des usagers (densité de production constante mais densité d'usagers croissante) :

$\left[\frac{\partial \ln C V(w, V, C A R)}{\partial \ln V}+\frac{\partial \ln C V(w, V, C A R)}{\partial \ln V_{p}}+\frac{\partial \ln C V(w, V, C A R)}{\partial \ln A b}\right]^{-1}$.

Enfin, les rendements d'échelle mesurent la variation des coûts variables moyens lorsque la production augmente avec le nombre d'abonnés, la taille du réseau Res (la quantité produite par usager et la densité d'usagers restent inchangées) et le nombre de communes Com. L'élasticité d'échelle s'écrit :

$\left[1-\frac{\partial \ln C V(w, V, C A R)}{\partial \ln R e s}\right]\left[\frac{\partial \ln C V(w, V, C A R)}{\partial \ln V}+\frac{\partial \ln C V(w, V, C A R)}{\partial \ln V_{p}}+\frac{\partial \ln C V(w, V, C A R)}{\partial \ln A b}+\frac{\partial \ln C V(w, V, C A R)}{\partial \ln C o m}\right]^{-1}$. Lorsque ces élasticités sont supérieures, égales ou inférieures à 1, les rendements sont respectivement croissants, constants ou décroissants.

Comme le montrent ces définitions, notre spécification (14) de la fonction de coût variable ne permet pas d'identifier directement les élasticités de coût par rapport au volume d'eau perdu, au nombre d'abonnés et à la longueur du réseau car nous utilisons à la place les variables d'indice linéaire de pertes et de densité. Il nous faut donc ré-agencer les termes de la fonction de coût 
translog. En simplifiant l'écriture translog aux termes premiers, on a :

$$
\ln (C V)=a_{0}+\sum_{h} a_{h} \ln w_{h}+a_{v} \ln V+b_{1} \ln I L P+b_{2} \ln A b+b_{3} \ln C o m .
$$

À partir de la définition de la variable $I L P$, une forme équivalente à cette équation est :

$$
\ln (C V)=a_{0}+\sum_{h} a_{h} \ln w_{h}+a_{v} \ln V+b_{1} \ln V_{p}-b_{1} \ln R e s+b_{2} \ln A b+b_{3} \ln C o m .
$$

Dans cette nouvelle relation, l'élasticité de coût par rapport à $V_{p}$ est égale à $b_{1}$, celle par rapport à $A b$ est égale à $b_{2}$ et l'élasticité de coût par rapport à Res est $-b_{1}$. Les résultats sur les différents rendements de réseau sont reportés dans le tableau 6 .

\begin{tabular}{|c|c|c|c|c|}
\hline Rendements de réseau & $\begin{array}{c}\text { Gestion } \\
\text { publique }\end{array}$ & & $\begin{array}{l}\text { Gestion } \\
\text { déléguée }\end{array}$ & \\
\hline Rendements de densité production & $\begin{array}{c}1,8352 \\
(0,1341)\end{array}$ & $* * *$ & $\begin{array}{c}1,4072 \\
(0,0659)\end{array}$ & $* * *$ \\
\hline Rendements de densité d'usagers & $\begin{array}{c}0,9912 \\
(0,0237)\end{array}$ & & $\begin{array}{c}0,9143 \\
(0,0322)\end{array}$ & $* * *$ \\
\hline Rendements d'échelle & $\begin{array}{c}0,9828 \\
(0,0130)\end{array}$ & & $\begin{array}{c}0,9814 \\
(0,0290)\end{array}$ & \\
\hline
\end{tabular}

TAB. 6 - Estimations des rendements de réseau

Notes : Rendements estimés pour le service moyen de chaque échantillon.

Écarts-types entre parenthèses calculés avec la méthode delta (KMENTA, [1986] ).

${ }^{* * *}$ : niveau de $1 \%$ pour le test de l'hypothèse $H_{0}$ : rendements $=1$.

Les tests d'hypothèse réalisés sur les différentes mesures de rendements de réseau nous donnent des indications sur les capacités d'accroitre la production pour le service moyen de chaque échantillon. Il existe des économies de densité de production significatives suggérant ainsi que les réseaux d'eau présentent une surcapacité afin de subvenir à une augmentation de la consommation des usagers. En outre, ces économies à exploiter sont plus importantes pour la gestion publique malgré une densité de production moyenne supérieure (environ $201 \mathrm{~m}^{3} / \mathrm{Ab}$ vs. $177 \mathrm{~m}^{3} / \mathrm{Ab}$ ). Par ailleurs, des rendements de densité d'usagers décroissants pour la gestion déléguée indiquent que le branchement de nouveaux usagers serait coûteux pour l'exploitant puisque les coûts variables moyens sont croissants avec le nombre d'usagers. En revanche, ces rendements sont constants dans le cas d'un service moyen en gestion publique présentant pourtant une densité d'abonnés très proche. Enfin, les résultats d'estimation indiquent des rendements d'échelle constants pour les deux modes de gestion, indiquant ainsi que le service moyen a atteint sa taille efficace 


\subsubsection{Mesure d'efficacité de coût}

Sur le modèle des équations (11) et (12), en supposant que la forme translog (14) a été choisie, nous écrivons la frontière de coût variable sous la forme simplifiée suivante :

$$
\ln C V_{i t}=a_{0}+\ln C V\left(R_{i t} ; \lambda\right)+\eta_{i}+\varepsilon_{c i t},
$$

où $i$ indice le service et $t$ la période de temps. $a_{0}$ est la constante de la régression à estimer en plus des paramètres $\lambda$ associés aux variables explicatives $R$ des coûts variables. $\eta_{i}$ et $\varepsilon_{c i t}$ sont définis dans la section 4.1. $\eta_{i}$ peut être estimé à partir des résidus de la régression de la façon suivante :

$$
\hat{\eta}_{i}=\frac{1}{T} \sum_{t}\left(\ln C V_{i t}-\hat{a}_{0}-\ln C V\left(R_{i t} ; \hat{\lambda}\right)\right)
$$

Mais une des propriétés de la mesure de l'efficacité de coût est qu'elle soit positive et inférieure ou égale à un (KumBHAKAR et LovelL, [2000]). On calcule alors $\hat{\eta}_{i}^{*}=\hat{\eta}_{i}-\min _{i}\left\{\hat{\eta}_{i}\right\} \geq 0$. L'efficacité de coût (ou efficacité productive) spécifique à l'exploitant est obtenue selon la formule suivante :

$$
C E_{i}=\exp \left(-\hat{\eta}_{i}^{*}\right)
$$

Selon cette définition, $C E_{i}$ est compris entre 0 et 1 . Sur l'échantillon étudié, l'exploitant le plus efficace correspond à $C E_{i}=1$, et les autres exploitants ont une efficacité $C E_{i}<1$.

TAB. 7 - Efficacité technique

\begin{tabular}{lcc}
\hline & $\begin{array}{c}\text { Gestion } \\
\text { publique } \\
(52 \text { services })\end{array}$ & $\begin{array}{c}\text { Gestion } \\
\text { déléguée } \\
(47 \text { services })\end{array}$ \\
\hline Moyenne & 0,6455 & 0,7478 \\
Médiane & 0,6205 & 0,7334 \\
Std & 0,0875 & 0,0818 \\
Min & 0,4736 & 0,6196 \\
Max & 1,0000 & 1,0000 \\
\hline
\end{tabular}

Les principales statistiques sur les valeurs d'efficacité estimées pour chaque mode de gestion sont reportées dans le tableau 7. Les valeurs estimées indiquent une efficacité supérieure pour les services en gestion déléguée avec des valeurs moyennes d'environ 0,65 pour les services d'eau potable en gestion publique et 0,75 en gestion déléguée. Un simple test de comparaison des moyennes ${ }^{31}$ conduit à rejeter l'hypothèse nulle d'égale efficacité. La différence d'efficacité entre les deux sous-échantillons est donc statistiquement significative bien que pas très importante. Dans la

\footnotetext{
${ }^{31}$ Sous l'hypothèse nulle d'égalité des moyennes empiriques $\bar{x}_{0}$ et $\bar{x}_{1}$, on peut utiliser une approximation normale
} 
mesure où gestion publique et gestion déléguée sont en concurrence et que les communes cherchent à optimiser (qualité vs. coûts) leur service d'AEP, on pouvait s'attendre à ce que les efficacités relatives des deux modes de gestion soient, à la marge, pas très différentes.

\section{$5 \quad$ Étude de la tarification}

À partir des coûts marginaux estimés dans la section précédente et toujours en tenant compte de la sélection du mode de gestion, l'objectif est maintenant d'identifier les facteurs qui justifient le niveau des tarifs observés. Dans ce but, nous modélisons le système de tarification de chaque mode de gestion et produisons l'analyse économétrique des équations économiques.

\subsection{Modélisation économique}

Dans le cas d'une gestion directe des services, la collectivité est supposée maximiser le surplus des usagers, net des coûts d'exploitation, sous contrainte d'équilibre budgétaire. Pour la gestion déléguée, un modèle avec information privée sur les coûts (BARON et MYERSON, [1982]) est considéré $^{32}$. L'objectif de la collectivité est de maximiser une somme pondérée des surplus des usagers et de l'exploitant, avec un poids plus élevé accordé aux usagers (dont l'intérêt doit être préservé en tant qu'électeurs des représentants municipaux). Le moyen utilisé par la collectivité pour atteindre cet objectif est de mettre en concurrence la position de monopole par le biais d'un appel d'offres.

\subsubsection{La demande des usagers}

La fonction de demande des usagers du service $i$ s'écrit $P_{i}^{g}\left(V_{i}^{g}, \varepsilon_{d i}^{g}\right)$ où $P_{i}^{g}$ est le prix unitaire pour le service $i$ en mode de gestion $g . \varepsilon_{d i}^{g}$ est une perturbation aléatoire de la demande, qui n'est pas connue de la collectivité ni de l'exploitant du service car elle capture des variables non observables expliquant la consommation des usagers. Comme dans la modélisation des coûts, on suppose que $\varepsilon_{d i}^{g}$ suit une loi normale; sa distribution s'écrit $N\left(0, \sigma_{\varepsilon_{d}^{g}}^{2}\right)$. Notons $S_{i}^{g}$ le surplus brut espéré des usagers :

$$
S_{i}^{g} \equiv S\left(V_{i}^{g}\right)=E\left[\int_{V_{i}^{g}}^{+\infty} P_{i}^{g}\left(x, \varepsilon_{d i}^{g}\right) d x\right]
$$

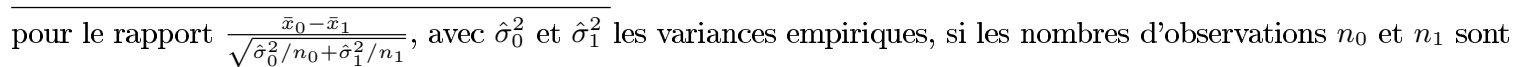
supérieurs à 30 .

${ }^{32}$ Comme WOLAK [1994], nous justifions ce choix plutôt qu'un modèle à la LAFFONT et TirOLE [1986] parce que les coûts d'exploitation ne font pas partie du contrat de délégation. Brocas et al. [2006] construisent leur modèle sur les mêmes hypothèses. 


\subsubsection{La gestion directe}

Dans le cas d'une gestion directe, la collectivité locale cherche à maximiser le bien-être social espéré (le surplus des usagers net des coûts espérés en gestion directe) sous contrainte budgétaire :

$$
\max _{V_{i}^{0}} S\left(V_{i}^{0}\right)-E\left[C V_{i}^{0}\left(R_{i}^{0}, \theta_{i}^{0}+\varepsilon_{c i}^{0}\right)+C F_{i}\right]
$$

telle que l'espérance de profit $\Pi_{i}^{0}$ de la collectivité locale ne soit pas négative :

$$
\Pi_{i}^{0} \equiv E\left[T_{i}^{0}+P_{i}^{0}\left(V_{i}^{0}, \varepsilon_{d i}^{0}\right) V_{i}^{0}-C V_{i}^{0}\left(R_{i}^{0}, \theta_{i}^{0}+\varepsilon_{c i}^{0}\right)-C F_{i}\right] \geq 0
$$

où $T_{i}^{0}$ représente la partie fixe du tarif pour le service $i$. Ce qui revient à maximiser :

$$
W_{i}^{0}=S\left(V_{i}^{0}\right)-E\left[C V_{i}^{0}\left(R_{i}^{0}, \theta_{i}^{0}+\varepsilon_{c i}^{0}\right)+C F_{i}\right]-\pi_{i}^{0} \Pi_{i}^{0},
$$

avec $\pi_{i}^{0} \geq 0$ représentant le coût de la contrainte budgétaire du service $i$.

Le prix optimal, solution de la maximisation de l'expression (20), est un prix Ramsey-Boiteux pour une régulation Cost of service ${ }^{33}$ :

$$
\frac{P_{i}^{0}-\partial C V_{i}^{0} / \partial V_{i}^{0}}{P_{i}^{0}}=\mu_{i}^{0} \frac{1}{\eta_{i}^{0}}
$$

où $\mu_{i}^{0}=\frac{\pi_{i}^{0}}{1+\pi_{i}^{0}}$ et $\eta_{i}^{0} \equiv E\left[\frac{d V / d P}{V_{i}^{0} / P_{i}^{0}}\right]$ est l'élasticité de la demande des usagers du service $i$.

Cette formule est la définition classique de l'indice de Lerner qui dépend de l'élasticité de la demande $\eta_{i}^{0}$ et du coût de la contrainte de budget $\pi_{i}^{0}$ du service $i$. On supposera que cette équation sous forme réduite est déterminée par les caractéristiques du service.

\subsubsection{La gestion déléguée}

Dans le cas d'une gestion déléguée, les préférences de la collectivité locale sont représentées par une somme pondérée du surplus net espéré $\left(U_{i}^{1}\right)$ des usagers du service $i$ et du profit espéré $\left(\Pi_{i}^{1}\right)$ tiré de l'exploitation du service $i$, avec $\pi^{1}$ le coefficient de pondération. Nous suivons l'approche de BARON et Myerson [1982] qui supposent que le surplus des usagers est préféré au profit de l'entreprise $\left.\left.\left(\pi^{1} \in\right] \frac{1}{2}, 1\right]\right)$. De la même manière, il n'y a pas de coût social des fonds publics dans notre modèle car le transfert monétaire $T_{i}^{1}$ entre la commune et l'entreprise ne fait pas appel à l'impôt. $T_{i}^{1}$ est la partie fixe du tarif binôme de l'eau directement prélevé par l'entreprise sur la facture de l'usager. L'entreprise $i$ a une information privée sur son efficacité productive (son type)

\footnotetext{
${ }^{33}$ Voir Laffont et TirOle, [1993], p.31.
} 
$\theta_{i}^{1}$. Nous supposons que $\theta_{i}^{1}$ est défini par la distribution $F($.$) , de densité f($.$) , sur l'intervalle \left[\underline{\theta}^{1}, \bar{\theta}^{1}\right]$, où $\underline{\theta}^{1}$ caractérise l'entreprise la plus efficace et $\bar{\theta}^{1}$ l'entreprise la moins efficace. La distribution est connue de la collectivité locale.

L'objectif de la collectivité locale est donc de maximiser l'expression suivante :

$$
\pi^{1} U_{i}^{1}+\left(1-\pi^{1}\right) \Pi_{i}^{1}
$$

où

$$
U_{i}^{1}=S\left(V_{i}^{1}\right)-E\left[P_{i}^{1}\left(V_{i}^{1}, \varepsilon_{d i}^{1}\right) V_{i}^{1}-T_{i}^{1}\right]
$$

et

$$
\Pi_{i}^{1}=E\left[T_{i}^{1}+P_{i}^{1}\left(V_{i}^{1}, \varepsilon_{d i}^{1}\right) V_{i}^{1}-C V_{i}^{1}\left(R_{i}^{1}, \theta_{i}^{1}+\varepsilon_{c i}^{1}\right)-C F_{i}\right]
$$

De façon équivalente, à partir des équations (23) et (24), nous pouvons réécrire : $U^{1}=S\left(V_{i}^{1}\right)-$ $\Pi_{i}^{1}-E\left[C V_{i}^{1}\left(R_{i}^{1}, \theta_{i}^{1}+\varepsilon_{c i}^{1}\right)+C F_{i}\right]$. En reportant ce résultat dans l'équation (22) et en divisant l'expression par $\pi^{1}$, le bien-être de la collectivité s'écrit finalement :

$$
W_{i}^{1}=S\left(V_{i}^{1}\right)-E\left[C V_{i}^{1}\left(R_{i}^{1}, \theta_{i}^{1}+\varepsilon_{c i}^{1}\right)+C F_{i}\right]-\mu^{1} \Pi_{i}^{1}
$$

avec $\left.\left.\mu^{1} \equiv \frac{2 \pi^{1}-1}{\pi^{1}} \in\right] 0,1\right]$.

Cette fonction du bien-être (en gestion déléguée) est à rapprocher de celle en gestion directe où seul le coefficient associé au profit du service doit être interprété différemment. Remarquons aussi que les expressions du bien-être dépendent crucialement des coûts engendrés lors de l'exploitation du service.

Puisque le paramètre d'efficacité $\theta_{i}^{1}$ n'est pas observé par la collectivité locale, elle maximise l'expression (25) sous la contrainte de participation $\left(\Pi^{1}\left(\theta^{1}\right) \geq 0, \forall \theta^{1}\right)$ et la contrainte d'incitation $\left(\Pi^{1}\left(\theta^{1}, \theta^{1}\right) \geq \Pi\left(\hat{\theta}^{1}, \theta^{1}\right), \forall \theta^{1}, \forall \hat{\theta}^{1}\right.$, où $\hat{\theta}^{1}$ est le type reporté par l'exploitant). Le mécanisme révélateur optimal donne le prix Baron-Myerson suivant :

$$
P_{i}^{1}-\frac{\partial C V_{i}^{1}}{\partial V_{i}^{1}}=\mu^{1} \frac{F\left(\theta_{i}^{1}\right)}{f\left(\theta_{i}^{1}\right)} \frac{\partial C V_{i}^{1}}{\partial \theta_{i}^{1} \partial V_{i}^{1}},
$$

où $F$ et $f$ représentent respectivement la fonction de distribution cumulative et la fonction de densité de $\theta_{i}^{1}$, et sont supposées connues de la collectivité locale. Si la collectivité locale organise une enchère pour attribuer le droit de servir la demande en eau potable pour le service $i$ dans le cas où l'entreprise a une information privée sur ses coûts, on obtient le prix Baron-Myerson pour l'entreprise sélectionnée, voir Mougeot et NAEGELEN [2005 $]^{34}$. La différence prix-coût marginal

\footnotetext{
${ }^{34}$ Le prix optimal défini par Mougeot et NAEgELEN [2005] est différent du nôtre parce qu'il intègre un coût
} 
dépend du paramètre $\theta^{1}$, du volume d'eau potable vendu (dépendant lui-même du paramètre $\left.\theta^{1}\right)^{35}$, des caractéristiques locales du service, de celles du contrat et de son environnement.

\subsection{Analyse économétrique}

\subsubsection{Procédure d'estimation}

Le modèle de tarification défini précédemment peut être affecté par le problème d'endogénéité du choix de mode de gestion. Les équations (21) et (26) peuvent donc se réécrire sous la forme économétrique suivante :

$$
\begin{aligned}
& M_{i t}^{0}=\beta^{M 0} X_{i t}^{M 0}+\gamma^{M 0} Z_{i}^{M 0}+\rho^{M 0}\left(\frac{\phi(\hat{\Psi})}{1-\Phi(\hat{\Psi})}\right)+\eta_{m i}^{0}+\epsilon_{m i t}^{0} \quad \text { si } u_{i} \leq-\lambda R_{i}, \\
& M_{i t}^{1}=\beta^{M 1} X_{i t}^{M 1}+\gamma^{M 1} Z_{i}^{M 1}+\rho^{M 1}\left(-\frac{\phi(\hat{\Psi})}{\Phi(\hat{\Psi})}\right)+\eta_{m i}^{1}+\epsilon_{m i t}^{1} \quad \text { si } u_{i}>-\lambda R_{i},
\end{aligned}
$$

où $i=1, \ldots, N_{0}$ et $t=1, \ldots, T_{0}$ pour le premier régime (gestion directe), et $i=1, \ldots, N_{1}$ et $t=$ $1, \ldots, T_{1}$ pour le second régime (gestion déléguée). $\epsilon_{m i t}^{0}$ et $\epsilon_{m i t}^{1}$ sont des termes d'erreur englobant les aléas non observés provenant des coûts et de la demande et sont respectivement supposés i.i.d $\left(0, \sigma_{\epsilon_{m}^{0}}\right)$ et $\left(0, \sigma_{\epsilon_{m}^{1}}\right) . \eta_{m i}^{0}$ et $\eta_{m i}^{1}$ sont des effets individuels ne dépendant pas du temps.

$M_{i t}^{0}$ représente l'indice de Lerner $\frac{P_{i t}^{0}-\partial C V_{i t}^{0} / \partial V_{i t}^{0}}{P_{i t}^{0}}$ et $M_{i t}^{1}$ la différence prix-coût marginal $P_{i t}^{1}-$ $\frac{\partial C V_{i t}^{1}}{\partial V_{i t}^{i}}$. Les coûts marginaux estimés $\widehat{C m}_{i t}^{0}$ et $\widehat{C m}_{i t}^{1}$ sont utilisés pour construire ces variables. Cela ajoute une erreur de mesure sur les variables dépendantes, mais elle est absorbée dans le terme d'erreur de la régression et peut être ignorée contrairement à une erreur de mesure sur une variable explicative, voir par exemple GREENE [2000].

Les variables $X_{i t}^{M 0}$ sont des variables variant dans le temps et $Z_{i}^{M 0}$ des variables invariant dans le temps. Les variables $X_{i t}^{M 1}$ et $Z_{i}^{M 1}$ sont définies de façon identique à celles du régime 0 . Elles incluent des variables du vecteur $R$ (variables explicatives des coûts variables) ${ }^{36}$ mais pas exclusivement. Dans le cas de la gestion déléguée, la durée du contrat ( $D U R E E$ ), le nombre d'avenants $(A V E N)$ et la concentration des opérateurs privés $(O P)$ peuvent être aussi des facteurs expliquant les variation de marge (voir l'annexe B pour la définition de ces nouvelles variables). Pour estimer les équation (27) et (28), les mêmes méthodes que celles décrites pour l'estimation des fonctions de coût (11) et (12) s'appliquent.

\footnotetext{
social des fonds public, que le régulateur est supposé utilitariste et que le coût marginal est supposé constant.

${ }^{35}$ Le principe du mécanisme révélateur est de faire dépendre les termes du contrat de l'information privée de l'exploitant. Dans le modèle plus complexe de Garcia et Thomas [2003], les volumes d'eau vendus et les volumes d'eau perdus font partie du contrat.

${ }^{36}$ Les marges sont régressées sur les termes de premier ordre du vecteur $R$ mais les termes quadratiques sont exclus.
} 


\subsubsection{Analyse descriptive des prix}

Notre base de données sur la tarification des services d'AEP permet de différencier le prix au $m^{3}$ d'eau consommée (part variable, Var) de la part fixe (Fixe) facturée à chaque abonné annuellement, mais aussi les montants des parts fixe et variable perçues par la collectivité dans le cas d'une gestion déléguée. Nous avons également calculé le prix moyen du $m^{3}$, noté $P_{R / V}$, comme la recette totale divisée par le volume d'eau facturé $V$. La recette est reconstituée à partir des parts variable et fixe du prix (y compris celles de la collectivité en gestion déléguée) $: P_{R / V}=$ $\frac{V a r \times V+\text { Fixe } \times A b}{V}$. On obtient ainsi un prix total pour le service d'eau potable. Le tableau 8 présente les statistiques descriptives des différents éléments du prix de l'eau potable (hors assainissement).

TAB. 8 - Statistiques descriptives sur les prix de l'eau potable

\begin{tabular}{|c|c|c|c|c|c|c|c|c|c|c|}
\hline \multirow[b]{2}{*}{ Prix } & \multirow[b]{2}{*}{ Part } & \multirow[b]{2}{*}{ Unité } & \multicolumn{4}{|c|}{$\begin{array}{l}\text { Gestion publique } \\
\text { (156 observations) }\end{array}$} & \multicolumn{4}{|c|}{$\begin{array}{c}\text { Gestion déléguée } \\
\text { (188 observations) }\end{array}$} \\
\hline & & & Moy & Std & Min & $\operatorname{Max}$ & Moy & Std & Min & Max \\
\hline Var & délégataire & $€ / m^{3}$ & & & & & 0,360 & 0,160 & 0,015 & 0,976 \\
\hline Var & collectivité & $€ / m^{3}$ & 0,702 & 0,196 & 0,305 & 1,265 & 0,267 & 0,149 & 0,00 & 0,678 \\
\hline Fixe & délégataire & $\epsilon$ & & & & & 26,78 & 12,74 & 9,15 & 65,89 \\
\hline Fixe & collectivité & $\epsilon$ & 50,86 & 20,60 & 10,46 & 140,32 & 17,56 & 10,17 & 0,00 & 49,09 \\
\hline$P_{R / V}$ & Total & $€ / m^{3}$ & 1,071 & 0,293 & 0,367 & 2,333 & 0,970 & 0,232 & 0,459 & 1,617 \\
\hline
\end{tabular}

Notes : Moy $=$ moyenne arithmétique, Std = écart-type.

Les parts variables facturées à l'usager sont en moyenne deux fois plus élevées pour les services en gestion publique $(0,70 €)$ que pour les services en gestion déléguée $(0,36 €)$. En particulier, la part variable moyenne en gestion publique est très proche de la moyenne des coûts variables moyens calculée, sur le sous-échantillon de services en régie, à près de $0,62 €$ (voir Tableau 1). Par ailleurs, la valeur moyenne de $P_{R / V}$ donne une idée plus juste du prix total du service d'eau potable. On peut ainsi constater que l'écart se réduit sensiblement (1,07€ vs. 0,97€) mais le prix reste supérieur pour la gestion publique. Or, les enquêtes au niveau national montrent toujours un prix de la délégation plus élevé. Il est donc important lors d'un comparaison des services de mettre en rapport les prix et les coûts marginaux. C'est ce que nous faisons dans la section suivante.

\subsubsection{Résultats d'estimation}

Comme pour l'estimation des équations de coût, nous devons valider dans un premier temps les hypothèses d'exogénéité. Nous réalisons un test d'Hausman de l'hypothèse nulle de non corrélation des effets individuels et des variables explicatives pour les deux équations de tarification. Dans le cas de la gestion publique, le test consistant à comparer l'estimateur VI-HT à l'estimateur des MCQG ne rejette pas l'hypothèse nulle, avec une valeur de la statistique égale à 4,72 et une p- 
value de 0,79. L'estimateur MCQG est donc un estimateur convergent et efficace. Dans le cas des services délégués, l'hypothèse nulle de non corrélation est rejetée avec une p-value de 0,0002. Nous considérons comme variables potentiellement corrélées avec les effets individuels le volume d'eau consommée $V$, l'indice de pertes $I L P$ et toutes les variables liées au contrat (DUREE, AVEN et $O P)$. Les autres variables sont utilisées comme instruments et entrent dans la composition de la matrice $A_{H T}$. L'utilisation des matrices $A_{A M}$ et $A_{B M S}$ est encore possible, elles apportent des gains d'efficacité. Le résultat du test d'Hausman (Within vs. VI-BMS) indique que l'hypothèse de validité des instruments n'est pas rejetée (avec une p-value égale à 0,99). Pour les service délégués, nous ferons donc nos commentaire sur la base des estimations VI-BMS.

Dans un premier temps, nous avons introduit dans le modèle pour la gestion déléguée le paramètre d'information privée $\theta_{i}$ ( $\eta_{i}$ en tenant compte de la variable de sélection) estimé ${ }^{37}$ à partir de l'équation (12) comme suggéré par l'équation structurelle (26) avec strictement les variables explicatives des coûts variables. Cependant, les variables du contrat comme le volume vendu dépendent théoriquement de ce paramètre. Ces termes sont donc corrélés et peuvent augmenter les problèmes de colinéarité. C'est ce que nous avons constaté avec des écarts-types élevés ${ }^{38}$. Les résultats d'estimation des équations ne prenant pas en compte (directement) le paramètre d'information privée sont présentés dans le tableau 9.

Pour les deux modes de gestion, les caractéristiques du réseau (et plus généralement les déterminants des coûts) sont des facteurs explicatifs des variations de marge observées. Pour les services en régie, un volume d'eau distribuée plus important (à nombre d'abonnés constant) donne une valeur plus grande de l'indice de Lerner. Cela semble indiquer que des services avec de gros consommateurs d'eau auront tendance à faire des marges plus importantes. Dans le même temps, le nombre d'abonnés fait baisser significativement ces marges. Les résultats nous apprennent aussi qu'un indice linéaire de perte plus bas conduit à des marges plus élevées. Un réseau en meilleur état, toutes choses égales par ailleurs, permet de distribuer de l'eau à moindre coût et donc entraîne mécaniquement une augmentation des marges si cela n'est pas répercuté dans le prix.

En gestion déléguée, volume et nombre d'abonnés n'ont pas d'impact direct significatif. Toutefois, comme pour les services en régie, un réseau de moins bonne qualité conduit à une différence prix-coût marginal plus faible. Nous avons également introduit dans la régression une variable appelée $O P$ indiquant la concentration de certains opérateurs privés sur le département. Son coefficient significativement négatif semble indiquer que les opérateurs les mieux implantés dans

\footnotetext{
${ }^{37}$ Introduire un résidu estimé issu d'une première régression, comme c'est le cas ici pour l'effet individuel, dans une seconde régression n'a pas d'impact sur l'efficacité de l'estimateur en deux étapes à condition que les régresseurs fassent partie de l'ensemble des variables expliquant la première régression, voir PAGAN [1984].

${ }^{38} \mathrm{Ce}$ terme d'inefficacité de coût n'était pas significatif dans le cas de la gestion publique, nous l'avons donc retiré de l'équation de tarification.
} 
TAB. 9 - Estimations du modèle de tarification

\begin{tabular}{|c|c|c|c|c|c|}
\hline \multirow[b]{2}{*}{ Variable } & \multicolumn{2}{|c|}{ Gestion publique } & \multicolumn{3}{|c|}{ Gestion déléguée } \\
\hline & Within & MCQG & Within & MCQG & BMS \\
\hline Constante & & $\begin{array}{c}0,7163^{* * *} \\
(0,0179)\end{array}$ & & $\begin{array}{c}0,1759^{* * *} \\
(0,0308)\end{array}$ & $\begin{array}{r}0,2955^{* *} \\
(0,1372)\end{array}$ \\
\hline $\ln A b$ & $\begin{array}{r}0,1459 \\
(0,4031)\end{array}$ & $\begin{array}{l}-0,1052^{* * *} \\
(0,0237)\end{array}$ & & & \\
\hline $\ln w_{E}$ & $\begin{array}{c}0,0667^{* * *} \\
(0,0175)\end{array}$ & $\begin{array}{c}0,0631^{* * *} \\
(0,0122)\end{array}$ & $\begin{array}{c}0,0324^{* * *} \\
(0,0132)\end{array}$ & $\begin{array}{c}0,0398^{* * *} \\
(0,0123)\end{array}$ & $\begin{array}{l}0,0330^{* * *} \\
(0,0131)\end{array}$ \\
\hline $\ln w_{T}$ & $\begin{array}{r}0,0070 \\
(0,0136)\end{array}$ & $\begin{array}{r}0,0180 \\
(0,0099)\end{array}$ & $\begin{array}{c}0,0312 \\
(0,0113)\end{array}$ & $\begin{array}{r}0,0241 \\
(0,0106)\end{array}$ & $\begin{array}{c}0,0312^{* * *} \\
(0,0112)\end{array}$ \\
\hline $\ln I L P$ & $\begin{array}{r}-0,0228 \\
(0,0139)\end{array}$ & $\begin{array}{l}-0,0419^{* * *} \\
(0,0081)\end{array}$ & $\begin{array}{l}-0,0778^{* * *} \\
(0,0309)\end{array}$ & $\begin{array}{l}-0,0838^{* * *} \\
(0,0247)\end{array}$ & $\begin{array}{l}-0,0815^{* * *} \\
(0,0303)\end{array}$ \\
\hline $\ln V$ & $\begin{array}{c}0,2049^{* * *} \\
(0,0614)\end{array}$ & $\begin{array}{c}0,2044^{* * *} \\
(0,0225)\end{array}$ & & & \\
\hline $\ln C o m$ & & $\begin{array}{r}-0,0276 \quad * \\
(0,0149)\end{array}$ & & $\begin{array}{r}-0,0214 \\
(0,0133)\end{array}$ & $\begin{array}{r}-0,0787 \quad * \\
(0,0451)\end{array}$ \\
\hline$O P$ & & & & $\begin{array}{l}-0,1987^{* * *} \\
(0,0370)\end{array}$ & $\begin{array}{l}-0,4635^{* * *} \\
(0,1666)\end{array}$ \\
\hline$O P \times \ln V$ & & & $\begin{array}{c}0,3077^{* * *} \\
(0,0521)\end{array}$ & $\begin{array}{c}0,1016^{* * *} \\
(0,0167)\end{array}$ & $\begin{array}{l}0,2716^{* * *} \\
(0,0454)\end{array}$ \\
\hline$O P \times \ln I L P$ & & & $\begin{array}{r}0,0735 \\
(0,0351)\end{array}$ & $\begin{array}{r}0,0558 \\
(0,0281)\end{array}$ & $\begin{array}{r}0,07166^{* *} \\
(0,0345)\end{array}$ \\
\hline Mill & & $\begin{array}{r}-0,0065 \\
(0,0164)\end{array}$ & & $\begin{array}{r}-0,0044 \\
(0,0137)\end{array}$ & $\begin{array}{r}-0,0024 \\
(0,0209)\end{array}$ \\
\hline Test d'Hausman & & $4,7241^{a}$ & & $23,837^{b}$ & $2,0419^{c}$ \\
\hline P-value & & 0,7866 & & 0,0002 & 0,9999 \\
\hline
\end{tabular}

la région réalisent des marges plus faibles. Cependant, ce résultat est atténué par les signes des coefficients de la variable $O P$ lorsqu'elle est croisée avec les variables $V$ et $I L P$. En particulier, lorsqu'un opérateur est bien implanté sur le département, l'état du réseau n'a pratiquement plus d'impact sur les marges : on passe de $-0,0815$ à $-0,0099(=-0,0815+0,0716)$. En outre, pour ces opérateurs, un volume d'eau plus élevé entraîne aussi une augmentation des marges. En revanche, les variables sur la durée de contrat ne semblent pas avoir d'effet significatif et ont été retirées de la régression.

Enfin pour les deux modes de gestion, plus le service regroupe de communes plus les marges sont basses. En effet, dans le cas d'une gestion déléguée, le rapport de force se rééquilibrant en faveur de la collectivité, cela permet de mieux négocier le prix de l'eau et donc de faire baisser les marges. En gestion publique, les prix sont tirés vers le bas par l'effet de concurrence entre les différents membres du syndicat.

Nous calculons maintenant à partir des paramètres estimés dans le tableau 9 les marges prédites pour les deux systèmes de tarification. Les statistiques descriptives sont reportées dans le 
tableau 10. Pour les services en gestion publique, les résultats valident l'hypothèse théorique de prix Ramsey-Boiteux en mettant en évidence des marges importantes et confirment l'observation faite lors de l'analyse des prix (section 5.2.2) selon laquelle la part variable du prix est plus proche du coût variable moyen que du coût marginal.

TAB. 10 - Estimation des marges

\begin{tabular}{lcc}
\hline & $\begin{array}{c}\text { Gestion } \\
\text { publique } \\
\text { (156 observations })\end{array}$ & $\begin{array}{c}\text { Gestion } \\
\text { déléguée } \\
\text { (188 observations })\end{array}$ \\
\hline Moyenne & 0,6539 & 0,0625 \\
Médiane & 0,6451 & 0,0637 \\
Std & 0,1058 & 0,2290 \\
Min & 0,3966 & $-0,4485$ \\
Max & 0,8789 & 0,5499 \\
\hline Notes : Pour la gestion publique, on calcule l'indice de \\
Lerner : $\left(\frac{P_{i t}^{0}-C m_{i t}^{0}}{P_{i t}^{0}}\right)$. Pour la gestion déléguée, on calcule \\
la différence prix-coût marginal $\left(P_{i t}^{1}-C m_{i t}^{1}\right)$.
\end{tabular}

En ce qui concerne les service délégués, les valeurs de l'écart prix-coût marginal peuvent être négatives certaines années. Cela corrobore les observations de résultats d'exercice négatifs faites sur les comptes-rendus financiers qui devraient pourtant en théorie représenter l'équilibre du contrat sur sa durée (CANNEva et al., [2004]). La valeur des marges est faible, en moyenne égale à $0,06 €$. Comme indiqué par l'équation (26), cette marge représente la rente informationnelle concédée par la collectivité locale à l'opérateur privé lors de la délégation du service. Un moyen de vérifier la pertinence du cadre d'analyse en information privée est de tester la significativité de cette rente. Nous testons donc l'hypothèse nulle $H_{0}:\left(\widehat{P_{i t}^{1}-C m_{i t}^{1}}\right)=0$ contre l'alternative unilatérale $H_{1}:\left(P_{i t}^{1} \widehat{-C m} m_{i t}^{1}\right)>0$. La valeur de la statistique de test est calculée par la formule $\frac{0,06}{\sqrt{0,23^{2} / 188}}=3,58$ et montre que l'hypothèse nulle est rejetée à un niveau de $1 \%$. Cela confirme donc la présence d'information privée et son impact sur les prix. Pour une moyenne de prix à $0,36 €$, on évalue la rente moyenne à environ $17 \%$.

\section{Conclusion}

La performance des services publics est une préoccupation centrale des autorités publiques. C'est également un thème de recherche important chez les économistes. Cependant, les travaux sur le secteur de l'eau proposent souvent des méthodologies qui ne prennent pas en compte simultanément le choix de mode de gestion des collectivités locales et son impact sur les coûts et les prix de l'eau, ce qui peut conduire à des résultats biaisés. Dans notre article, nous modélisons 
les interactions entre le mode de gestion des services d'AEP et leurs coûts d'exploitation avant d'estimer leur efficacité et leur coût marginal. Nous cherchons ensuite les facteurs expliquant les niveaux observés des tarifs de chaque mode de gestion.

Un modèle de régimes est développé sur la base des deux modes de gestion existants en France : la gestion directe publique et la gestion déléguée. Le choix de la collectivité locale est supposé dépendre de la différence de coûts d'exploitation selon le mode de gestion et des caractéristiques du service (taille, état du réseau, etc.) . Le mode de tarification est ensuite modélisé. La maximisation du bien-être social espéré conduit à un prix Ramsey-Boiteux en gestion directe et un prix Baron-Myerson en gestion déléguée. Afin de valider empiriquement ces modèles, nous utilisons une procédure corrigeant les possibles biais de sélection (LEE, [1978]), adaptée aux données de panel, pour estimer les équations de coût et de tarification.

Les résultats d'estimation confirment l'existence de biais de sélection liés au choix de mode gestion dans l'estimation des fonctions de coût. L'équation structurelle de choix entre gestion directe et gestion déléguée offre un très bon ajustement aux données de services d'AEP avec un $R^{2}$ de 0,74 et $94 \%$ de bonnes prédictions du mode de gestion. La comparaison des coûts est l'un des principaux déterminants du choix de la collectivité locale. Ce choix est aussi dicté par les caractéristiques du service et le mode de gestion du service d'assainissement.

À partir des paramètres estimés des fonctions de coût, la structure de panel de nos données nous permet d'estimer l'effet individuel invariant dans le temps représentant l'efficacité de l'exploitant. Le taux moyen d'efficacité s'élève à 0,75 pour les services délégués contre 0,65 pour les services en régie. Cette différence entre les deux modes de gestion est statistiquement significative. Concernant la performance de réseau, les économies de densité de production réalisables pour tous les services mettent en évidence les capacités des infrastructures à répondre à une augmentation importante de la consommation des usagers. Par ailleurs, le service moyen d'AEP de chaque sous-échantillon a atteint sa taille efficace en exploitant au maximum ses économies d'échelle par le regroupement de communes. Cependant, les rendements de densité d'usagers sont décroissants pour les services en gestion déléguée. Cela indique que le branchement de nouveaux usagers est coûteux pour l'exploitant. Le coût moyen est en revanche constant pour le service moyen en régie, semblant ainsi montrer une meilleure gestion du réseau d'eau.

Nous estimons ensuite les équations de tarification en introduisant les coûts marginaux calculés dans l'analyse économétrique des coûts. Les caractéristiques des services expliquent aussi le niveau des prix et des marges. Par exemple, un réseau en meilleur état physique entraîne une augmentation des marges (récompensant les efforts des exploitants) alors qu'un service regroupant un plus grand nombre de communes les fait baisser. Dans le cas de la gestion déléguée, la concentration des opérateurs marque un effet plus important sur les rentes des variables inclues dans le contrat 
telles que le volume d'eau pour les usagers et l'indice de pertes. Par exemple, la qualité du réseau n'a quasiment plus d'impact sur les marges lorsque le contrat a été signé par un opérateur privé bien implanté régionalement. Enfin, le calcul des marges prédites nous permet de valider le cadre d'asymétrie d'information de la gestion déléguée. 


\section{Références}

Amemiya T. ET MaCuRdy T. (1986). - « Instrumental-variable estimation of an errorcomponents model », Econometrica, 54(4), p. 869-880.

Baron D. P. et Myerson R. B. (1982). - « Regulating a monopolist with unknown costs », Econometrica, 50(4), p. 911-930.

Bhattacharyya A., Harris T. R., Narayanan R. et Raffiee K. (1995). - « Specification and estimation of the effect of ownership on the economic efficiency of the water utilities », Regional Science and Urban Economics, 25(6), p. 759-784.

Boumahdi R. eT Thomas A. (1992). - « Estimation des modèles à deux régimes avec des données de panel », Annales d'Économie et de Statistique, 28, p. 125-142.

Breusch T., Mizon G. et Schmidt P. (1989). - « Efficient estimation using panel data », Econometrica, 57(3), p. 695-700.

Brocas I., Chan K. et Perrigne I. (2006). - « Regulation under asymmetric information in water utilities », American Economic Review : Papers and Proceedings, 96(2), p. 62-66.

Canneva G., Garcia S. et Guérin-Schneider L. (2004). - « La production des comptes dans les contrats d'eau et d'assainissement : Outil de régulation pour la collectivité ou de négociation pour le délégataire », Cahiers de recherche du GEA, Cahier No 2 - Septembre.

Carpentier A., Nauges C., Reynaud A. et Thomas A. (2006). - « Effets de la délégation sur le prix de l'eau potable en france : Une analyse à partir de la littérature sur les « effets de traitement »», Économie et Prévision, 178(3), p. 1-19.

Chong E., Huet F., Saussier S. et Steiner F. (2006). - « Public-private partnerships and prices : Evidence from water distribution in france », Review of Industrial Organization, 29(1), p. 149-169.

Christensen L. R., Jorgenson D. W. et Lau L. J. (1971). - « Conjugate duality and the transcendental logarithmic production function », Econometrica, 39(4), p. 255-256.

COUR DES COMPTES (1997). - « La gestion des services publics locaux d'eau et d'assainissement », Rapport Public Particulier, $n^{o}$ 4327, Les éditions du Journal Officiel.

(2003). - « La gestion des services publics locaux d'eau et d'assainissement », Rapport Public Particulier, $n^{\circ}$ 4466, Les éditions des Journaux Officiels.

Crain W. M. et ZardKoohi A. (1978). - « A test of the property-rights theory of the firm : Water utilities in the united states », Journal of Law and Economics, 21(2), p. 395-408.

DDAF GiRonde $(1995,1996,1997,1998)$. - « Rapports techniques et financiers sur les services d'eau potable délégués en gironde », Documents Internes. 
Demsetz H. (1968). - «Why regulate utilities?», Journal of Law and Economics, 11(1), p. $55-65$.

Diewert W. E. ET WAles T. J. (1987). - «Flexible functional forms and global curvature conditions », Econometrica, 55(1), p. 43-68.

Estache A. et Rossi M. A. (2002). - « How different is the efficiency of public and private water companies in asia?», World Bank Economic Review, 16(1), p. 139-148.

Feigenbaum S. et Teeples R. (1983). - « Public versus private water delivery : a hedonic cost approach », Review of Economics and Statistics, 64, p. 672-678.

Garcia S. et Thomas A. (2001). - « The structure of municipal water supply costs : Application to a panel of french local communities », Journal of Productivity Analysis, 16(1), p. 5-29.

(2003). - « Regulation of public utilities under asymmetric information : The case of municipal water supply in france », Environmental and Resource Economics, 26(1), p. 145-162.

Greene W. H. (2000). Econometric analysis. Prentice Hall International, Inc., fourth edn.

GuÉRIN-Schneider L. ET NAKHLA M. (2003). - « Les indicateurs de performance : Une évolution clef dans la gestion et la régulation des services d'eau et d'assainissement », Flux, 52/53, p. $55-68$.

Hausman J. (1978). - « Specification tests in econometrics », Econometrica, 46(6), p. 1251-1271.

Hausman J. et TAYlor W. (1981). - « Panel data and unobservable individual effects », Econometrica, 49(6), p. 1377-1398.

HeCKMAn J. J. (1976). - « The common structure of statistical models of truncation, sample selection, and limited dependant variables and a simple estimator for such models », The Annals of Economic and Social Measurement, 5(4), p. 475-492.

(1979). - « Sample selection bias as a specification error », Econometrica, 47(1), p. $153-161$.

Huang M.-Y., Huang C. J. ET Fu T.-T. (2002). - « Cultivation arrangements and the cost efficiency of rice farming in taiwan », Journal of Productivity Analysis, 18(3), p. 223-239.

IfEN (2001). - « Eau potable : Diversité des services... grand Écart des prix », sous la direction de Gisèle Rebeix. Les données de l'environnement - eau, $n^{\circ} 65$.

Kmenta J. (1986). Elements of econometrics. Macmillan Publishing Company, New York, second edn.

Kumbhakar S. C. ET Lovell C. A. K. (2000). Stochastic frontier analysis. Cambridge University Press, Cambridge, UK. 
LAfFont J.-J. ET Tirole J. (1986). - « Using cost observation to regulate firms », Journal of Political Economy, 94(3), p. 614-641.

(1993). A theory of incentives in procurement and regulation. MIT Press, Cambridge, Massachussets.

LEE L.-F. (1978). - « Unionism and wage rates : A simultaneous equations model with qualitative and limited dependent variables », International Economic Review, 19(2), p. 415-433.

Lee L.-F., Maddala G. S. Et Trost R. P. (1980). - « Asymptotic covariance matrices of twostage probit and two-stage tobit methods for simultaneous equations models with selectivity », Econometrica, 48(2), p. 491-503.

Mougeot M. et Naegelen F. (2005). - « La concurrence pour le marché », Revue d'Économie Publique, 115(6), p. 739-778.

Ménard C. ET Saussier S. (2000). - « Contractual choice and performance : the case of water supply in france », Revue d'Économie Industrielle, 92, p. 385-404.

PAGAN A. (1984). - « Econometric issues in the analysis of regressions with generated regressors », International Economic Review, 25(1), p. 221-247.

Reynaud A. et Thomas A. (2005). - « Demande domestique en eau et choix de délégation : le cas français », Revue Économique, 56(5), p. 1145-1168.

Riordan M. H. et Sappington D. E. M. (1987). - « Awarding monopoly franchises », American Economic Review, 77(3), p. 375-387.

SAAL D. S. ET PARKer D. (2000). - « The impact of privatization and regulation on the water and sewerage industry in england and wales : A translog cost function model », Managerial and Decision Economics, 21(6), p. 253-268.

SAGE E. (1999). - « La concurrence par comparaison ("yardstick competition") : Théorie et applications. une proposition pour le secteur de l'eau en france », Thèse de Doctorat, Université Paris IX - Dauphine.

Sevestre P. (2002). Économétrie des données de panel. Dunod, Paris.

TAVERniER Y. (2001). - « Le financement et la gestion de l'eau », Rapport d'Information, $n^{o} 3081$, Assemblée Nationale.

Teeples R. et Glyen D. (1987). - « Cost of water delivery systems : Specification and ownership effects », Review of Economics and Statistics, 69, p. 399-407.

WOLAK F. A. (1994). - « An econometric analysis of the asymmetric information, regulator-utility interaction », Annales d'Économie et de Statistique, 34, p. 13-69. 


\section{Annexes}

\section{A Estimation des variances des estimateurs}

Dans ce qui suit, afin de gagner de la place, on présente les variances des estimateurs pour le régime $j$. Lorsque cela est nécessaire, on utilise les indices ' 0 ' et ' 1 ' pour différencier les régimes.

\section{A.1 Estimateur Within}

La matrice de variance-covariance de l'estimateur Within $\hat{\beta}_{Q}^{j}$ s'écrit :

$$
\operatorname{Var}\left(\hat{\beta}_{Q}^{j}\right)=\hat{\sigma}_{\varepsilon^{j}}^{2}\left(X^{j^{\prime}} Q X^{j}\right)^{-1}
$$

L'estimation Within élimine les effets individuels dans les équations à erreurs composées. De cette façon, il n'y a plus de corrélation entre ces termes et certaines variables explicatives. L'estimateur convergent de $\sigma_{\epsilon j}^{2}$ proposé est donc, voir SEVESTRE [2002], p.26 :

$$
\hat{\sigma}_{\varepsilon^{j}}^{2}=\frac{\hat{\varepsilon}_{Q^{j}}^{j^{\prime}} \hat{\varepsilon}_{Q}^{j}}{n_{j}\left(t_{j}-1\right)-k_{j}^{q}},
$$

où $\hat{\varepsilon}_{Q}^{j}=Q C V^{j}-Q X^{j} \hat{\beta}_{Q}^{j}$ sont les résidus de l'estimation Within et $k_{j}^{q}$ le nombre de variables $X^{j}$.

\section{A.2 Estimateur MCQG}

La matrice de variance-covariance de l'estimateur $\hat{\Gamma}_{M C Q G}$ est donnée par la formule suivante, voir Boumahdi et Thomas [1992] :

$$
V\left(\hat{\Gamma}_{M C Q G}^{j}\right)=\hat{\sigma}_{\epsilon^{j}}^{2}\left(\widetilde{H}^{j^{\prime}} \widetilde{H}^{j}\right)^{-1}-\hat{\rho}^{j 2}\left(\widetilde{H}^{j^{\prime}} \widetilde{H}^{j}\right)^{-1} \widetilde{H}^{j^{\prime}}\left[C^{j}-C^{j} W^{j}\left(W^{\prime} \Lambda W\right)^{-1} W^{j^{\prime}} C^{j}\right] \widetilde{H}^{j}\left(\widetilde{H}^{j^{\prime}} \widetilde{H}^{j}\right)^{-1}
$$

Afin de construire cette matrice, nous devons définir les matrices diagonales $C^{j}$ et $\Lambda$ établies à partir des ratios de Mills inverses, mais aussi rappeler la matrice des $h$ variables explicatives $R$ du modèle de sélection, de dimension $\left(N_{0} T_{0}+N_{1} T_{1}\right) \times h$ :

$$
R=\left[R^{0}, R^{1}\right]=\left[r_{1,1}^{0} \ldots r_{1, T_{0}}^{0}, \ldots, r_{N_{0}, 1}^{0} \ldots r_{N_{0}, T_{0}}^{0}, r_{N_{0}+1,1}^{1} \ldots r_{N_{0}+1, T_{1}}^{1}, \ldots, r_{N_{0}+N_{1}, 1}^{1} \ldots r_{N_{0}+N_{1}, T_{1}}^{1}\right]
$$


La matrice $C^{0}$ est définie comme suit :

$$
C^{0}=\left[\begin{array}{cccc}
\left(\frac{\phi_{1}}{1-\Phi_{1}}\right)^{2}-r_{1}^{0} \lambda \frac{\phi_{1}}{1-\Phi_{1}} & 0 & \cdots & 0 \\
0 & \left(\frac{\phi_{2}}{1-\Phi_{2}}\right)^{2}-r_{2}^{0} \lambda \frac{\phi_{2}}{1-\Phi_{2}} & \cdots & 0 \\
\vdots & \cdots & \ddots & \left(\frac{\phi_{N_{0}}}{1-\Phi_{N_{0}}}\right)^{2}-r_{N_{0}}^{0} \lambda \frac{\phi_{N_{0}}}{1-\Phi_{N_{0}}}
\end{array}\right] \otimes I_{T_{0}}
$$

La matrice $C^{1}$ a pour terme diagonal $r_{i}^{1} \lambda \phi_{i} / \Phi_{i}+\left(\phi_{i} / \Phi_{i}\right)^{2}$, pour $i=N_{0}+1, \ldots, N_{0}+N_{1}$. Elle est ensuite reproduite sur $T_{1}$ périodes de temps de façon similaire à $C^{0}$. Par ailleurs, $\Lambda$ s'écrit :

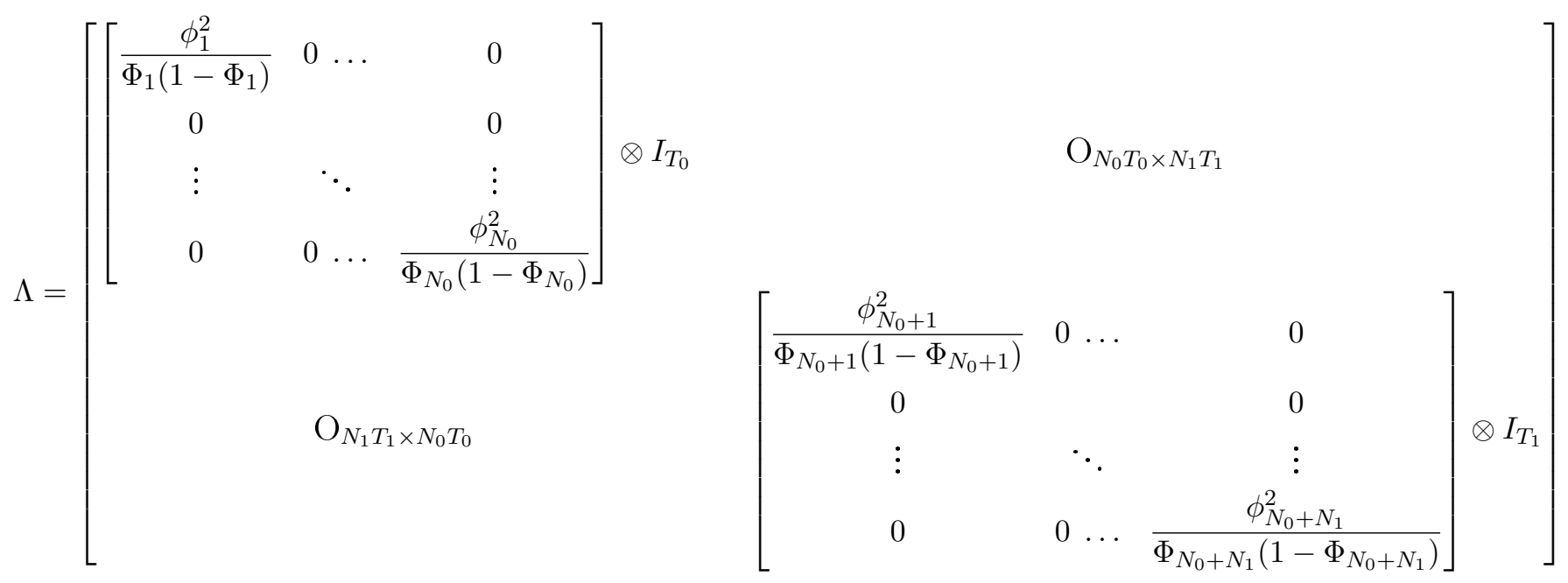

Pour chaque observation $i$ des deux régimes, les termes diagonaux sont le produit des ratios de Mills inverses dans les cas $j=0$ et $j=1$. Parce que nous disposons de données de panel, la matrice doit être adaptée en reproduisant chaque terme sur le nombre d'années observées. De plus, comme notre échantillon n'est pas cylindré, nous appliquons aux termes de chaque régime une matrice identité de dimension différente correspondant au nombre d'années observées pour chaque régime. De plus, $\widetilde{H}^{j}$ est fonction de $\theta$, où $\theta=\sigma_{\epsilon^{j}}^{2} / \bar{\sigma}^{j 2}$ avec $\bar{\sigma}^{j 2}=\sigma_{\eta^{j}}^{2}+\sigma_{\epsilon^{j}}^{2} / t^{j}$. On a donc besoin d'un estimateur convergent de $\theta$. Pour cela, on utilise l'estimateur donné par l'équation (A.1) pour estimer $\sigma_{\epsilon^{j}}^{2}$. En outre, un estimateur convergent de $\bar{\sigma}^{j 2}$ est, selon SEVESTRE [2002], p.26 :

$$
\hat{\bar{\sigma}}^{j 2}=\frac{\hat{v}_{B}^{j^{\prime}} \hat{v}_{B}^{j}}{t_{j}\left(n_{j}-k_{j}^{b}\right)},
$$

où $\hat{v}_{B}^{j}$ sont les résidus Between estimés (sur $n_{j} \times t_{j}$ observations) de la façon suivante $: \hat{v}_{B}^{j}=$ $B C V^{j}-B X^{j} \hat{\beta}_{B}^{j}-B Z^{j} \hat{\gamma}_{B}^{j}-B M i l l^{j} \hat{\rho}_{B}^{j}$, avec $\left[\hat{\beta}_{B}^{j}, \hat{\gamma}_{B}^{j}, \hat{\rho}_{B}^{j}\right]$ les estimateurs Between et $k_{j}^{b}$ le nombre total de paramètres estimés. 


\section{A.3 Estimateur VI}

La matrice de variance-covariance de l'estimateur $\hat{\Gamma}_{V I}^{j}$ est :

$V\left(\hat{\Gamma}_{V I}^{j}\right)=\hat{\sigma}_{\epsilon^{j}}^{2}\left(\widetilde{H}^{j^{\prime}} P_{A}^{j} \widetilde{H}^{j}\right)^{-1}-\hat{\rho}^{j 2}\left(\widetilde{H}^{j^{\prime}} P_{A}^{j} \widetilde{H}^{j}\right)^{-1} \widetilde{H}^{j^{\prime}} P_{A}^{j}\left[C^{j}-C^{j} W^{j}\left(W^{\prime} \Lambda W\right)^{-1} W^{j^{\prime}} C^{j}\right] P_{A}^{j} \widetilde{H}^{j}\left(\widetilde{H}^{j^{\prime}} P_{A}^{j} \widetilde{H}^{j}\right)^{-1}$

On utilise encore l'estimateur convergent de $\sigma_{\epsilon^{j}}^{2}$ donné par la formule (A.1). En revanche, pour l'estimation de $\bar{\sigma}^{j 2}$, on doit procéder d'une manière différente du cas des MCQG parce qu'il y a le problème d'endogénéité de certaines variables. On calcule d'abord les résidus Within sur les variables $X^{j}$ transformées par l'opérateur Between $: h^{j}=B C V^{j}-B X^{j} \hat{\beta}_{Q}^{j}$. Ces résidus sont ensuite régressés sur les variables invariant dans les temps $Z^{j}$ tout en prenant en compte les ratios de Mills inverses. Ainsi, l'estimateur de $\gamma_{Z}^{j}$ est donné par la méthode des doubles moindres carrés, voir Boumahdi et Thomas [1992] $: \hat{\gamma}_{Z}^{j}=\left(Z^{\prime j} P_{A}\left(I_{n_{j} t_{j}}-P_{M}\right) P_{A} Z^{j}\right) Z^{\prime j} P_{A}\left(I_{n_{j} t_{j}}-P_{M}\right) P_{A} d_{i}$, où $A^{j}$ est une matrice d'instruments (on prendra ici les variables supposés exogènes $X_{1}^{j}$ et $Z_{1}^{j}$ ), $P_{A}$ sa matrice de projection et $P_{M}$ la matrice de projection de la variable $M i l l^{j}$ pour le régime $j$. Finalement, dans l'équation (A.2) de l'estimateur de $\bar{\sigma}^{j 2}$, les résidus Between $\hat{v}_{B}^{j}$ sont remplacés par les résidus suivants :

$$
B C V^{j}-B X^{j} \hat{\beta}_{Q}^{j}-B Z^{j} \hat{\gamma}_{Z}^{j}
$$

\section{B Variables de contrat en gestion déléguée}

Plusieurs données de contrat dans le cas d'une gestion déléguée peuvent avoir une influence sur le niveau des prix et des marges. En particulier, on s'attend à ce qu'un contrat plus long, et donc une concurrence plus faible, ait un impact positif sur les marges. La durée de contrat (DUREE) et le nombre d'avenants $(A V E N)$ mesurent cet effet. De façon similaire, une forte concentration d'opérateurs privés diminue la concurrence et peut aussi affecter les marges à la hausse. La variable binaire $O P$ est égale à un lorsque l'opérateur exploitant le service est l'un des deux opérateurs les plus présents sur le département. Les statistiques descriptives pour ces variables sont reportées dans le tableau B.1.

TAB. B.1 - Statistiques descriptives sur les variables de contrat

\begin{tabular}{llrrrr}
\hline Variable & Définition & Moy & Std & Min & Max \\
\hline DUREE & Durée de contrat (nombre d'années) & 16,57 & 7,13 & 6,00 & 42,00 \\
$A V E N$ & Nombre d'avenants au contrat & 0,26 & 0,61 & 0,00 & 2,00 \\
$O P$ & $=1$ : opérateur privé le plus présent dans le département & 0,83 & 0,38 & 0,00 & 1,00 \\
\hline
\end{tabular}

Notes : Nombre de services $=47$. Moy $=$ moyenne arithmétique, Std $=$ écart-type. 